\title{
RECENTI SVILUPPI NELLA RISONANZA MAGNETICA NUCLEARE A PAVIA
}

\author{
MARTA FILIBIAN $(*)$ e PIETRO CARRETTA $(* *)$
}

Nota presentata dal m.e. Attilio Rigamonti

(Adunanza del 12 novembre 2020)

SunTO. - La Risonanza Magnetica Nucleare (NMR) è una tecnica sperimentale cruciale per lo studio della materia in numerosi ambiti e applicazioni, solo a titolo di esempio nella fisica, nella chimica, nella scienza dei materiali, nella biologia, nella medicina e nella scienza dell'alimentazione. Con l'utilizzo di questa tecnica, il gruppo di ricerca NMR dell'Università degli Studi di Pavia (UniPV) ha fornito importanti contributi scientifici nei settori della Fisica della Materia e della Fisica Applicata al settore Biomedico. In questa nota verranno illustrati alcuni dei risultati più rilevanti che $i$ ricercatori afferenti al gruppo hanno prodotto nel corso di decenni di incessante ed appassionato lavoro, con esempi riguardanti lo studio dei Magneti Molecolari, dei Superconduttori ad alta Temperatura, degli agenti di contrasto e dell'iperpolarizzazione di molecole per la Risonanza Magnetica per Immagini. Verranno anche riportati alcuni risultati preliminari ottenuti dal Centro di Imaging Preclinico di UniPV, fondato recentemente con il contributo fondamentale del gruppo NMR.

$* * *$

ABSTRACT. - Nuclear Magnetic Resonance (NMR) is a crucial experimental technique for the study of matter in several fields and applications, as for example in physics, chemistry, materials science, biology, medicine and food science. By means of this technique, the NMR group of the University of Pavia (UniPV) has given valuable scientific contributions to the Condensed Matter Physics and to the Physics applied to the Biomedical sector. In this note we'll illustrate some of the most relevant results produced by the researchers of

(") Università degli Studi di Pavia, Centro Grandi Strumenti, Pavia, Italy. E-mail: marta.filibian@unipv.it

(**) Università degli Studi di Pavia, Dipartimento di Fisica, Pavia, Italy. E-mail: pietro.carretta@unipv.it 
the group within decades of work carried out with passion and dedication, with examples regarding the Molecular Magnets, the High Tc Superconductors, the contrast agents and the hyperpolarization of molecules for Magnetic Resonance Imaging. We'll also report some preliminary results obtained at the Preclinical Imaging Facility of UniPV, recently founded with the key contribution of the NMR group.

\section{INTRODUZIONE}

La Risonanza Magnetica Nucleare, in inglese Nuclear Magnetic Resonance (NMR), è una tecnica sperimentale di indagine della materia che tutt'oggi ha un ruolo di grande rilievo in un numerosi ambiti e applicazioni, solo a titolo di esempio nella fisica, nella chimica, nella scienza dei materiali, nella biologia, nella medicina e nella scienza dell'alimentazione. Essa si basa sul magnetismo nucleare e sfrutta la possibilità di manipolare l'orientazione della magnetizzazione nucleare generata da un campo magnetico irraggiando ad una precisa frequenza, detta di risonanza, nel dominio delle Radiofrequenze (RF). Il segnale NMR è ricco di informazioni caratteristiche, poiché i nuclei sono vere e proprie sonde locali naturalmente presenti nei materiali, capaci di rilevare i fenomeni fisici microscopici in atto nel loro intorno.

La realizzazione dei primi esperimenti di NMR è datata agli anni '40. Sono trascorsi, quindi, circa 80 anni dalla nascita della NMR, durante i quali essa è diventata uno strumento di indagine sperimentale estremamente potente e raffinato. Il gruppo di ricerca NMR dell'Università degli Studi di Pavia ha dato un importante contributo nei numerosi ambiti di ricerca correlati all'uso di questa tecnica, principalmente nella Fisica della Materia e nella Fisica Applicata al settore Biomedico, sin dalla sua nascita. In questa nota verranno illustrati alcuni dei risultati più rilevanti che i ricercatori afferenti al gruppo hanno prodotto nel corso di decenni di incessante ed appassionato lavoro e che hanno condotto a progressi in studi di carattere sia sperimentale che teorico, grazie a prestigiose collaborazioni nazionali ed internazionali nel settore accademico ed industriale.

\subsection{La nascita della NMR tra America ed Europa ed i primi esperimenti a Pavia}

I primi esperimenti di NMR, inizialmente orientati alla misurazione dei momenti magnetici di spin dei nuclei, furono condotti sul 
finire degli anni '30 e nel corso degli anni '40 da ricercatori sia Statunitensi che Europei. Nel 1938 Isidor Rabi, premio Nobel nel 1944, fu in grado di misurare la condizione di risonanza magnetica in un fascio di molecole di LiCl alla Columbia University (Rabi 1938). L'idea sperimentale si rifaceva all'esperimento di Stern su fasci di idrogeno molecolare e giungeva dopo alcuni risultati negativi dell'olandese Gorter (Gorter 1942) che aveva provato a rilevare la risonanza in cristalli puri ed a basse temperature con metodi calorimetrici. Successivamente, nel 1946 Purcell e Bloch, premi Nobel nel 1952, pubblicarono due lavori indipendenti a distanza di poche settimane, in cui riportavano il fenomeno della risonanza nella materia condensata, l'uno nella paraffina, l'altro nell'acqua. L'esperimento di Bloch a Stanford (Bloch 1946) lasciava già intravedere come si sarebbe evoluta la strumentazione per NMR nei decenni successivi ed era conseguenza degli avanzamenti tecnologici avvenuti durante la Seconda Guerra Mondiale nel campo delle tecnologie Radar. Una fialetta d'acqua era sistemata al centro di due bobine di trasmissione e ricezione con assi tra loro perpendicolari, accordate ad una frequenza fissa di alcuni $\mathrm{MHz}$. Le bobine ed il campione erano a loro volta posizionate in un elettromagnete, con entrambi gli assi perpendicolari al suo campo. Il campo dell'elettromagnete veniva quindi variato lentamente fino ad ottenere la risonanza dei nuclei di idrogeno dell'acqua. Il riorientamento dei momenti di spin nucleari causato dalla RF trasmessa al campione era rilevato sotto forma di segnale elettrico dalla bobina di ricezione tramite il fenomeno dell'induzione elettromagnetica, secondo la legge di Faraday.

Negli stessi anni in Europa venivano condotti altri esperimenti indipendenti basati sulla ricezione radio della "induzione nucleare", come definito da Bloch. In particolare nel 1946 Luigi Giulotto, venuto a conoscenza degli esperimenti condotti da Bloch e Purcell, decise di costruire il primo apparato europeo per NMR a Pavia, partendo da materiali di facile reperibilità. Grazie all'esperienza nelle radiotrasmissioni acquisita durante la Guerra e dopo aver acquistato un ricevitore radio alla fiera di Senigallia, riuscì ad installare e ad ascoltare il primo segnale NMR. A differenza di Bloch, non avendo un oscilloscopio, egli aveva deciso di rilevare il segnale di induzione nucleare al variare del campo magnetico acusticamente, utilizzando una cuffia. Una volta egli disse: "Potevo ascoltare il segnale perché avevo la fede". Nel 1947 Giulotto riuscì ad acquistare un oscilloscopio e assieme a Gigli vide i 
primi segnali NMR dei protoni dell'acqua (Fig. 1) ed iniziarono i primi studi sugli effetti dei tempi di rilassamento. All'inizio degli anni '50 viene costruito un nuovo spettrometro che ha permesso di condurre studi sull'inversione della popolazione statistica, sul rilassamento nei liquidi con impurezze paramagnetiche, nei metalli liquidi e di iniziare a studiare $i$ processi di rilassamento dovuti alla modulazione dell'interazione quadrupolare. Viene inoltre ideato un nuovo metodo per misurare il $T_{2}$.

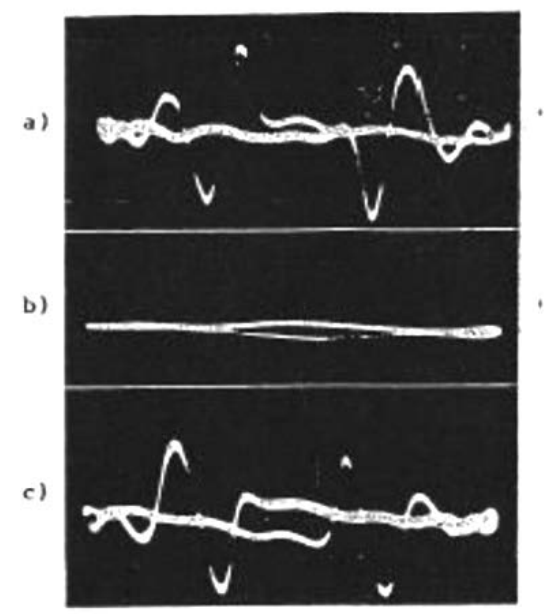

Fig. 1. Primi segnali NMR del protone nell'acqua al variare dell'intensità del campo magnetico.

Dopo i lavori di Hahn sull' "eco di spin", a partire dagli anni '50 (Hahn 1950), vi fu un progressivo passaggio verso esperimenti e strumentazione che permettesse l'utilizzo di impulsi a RF. Nel 1965 iniziano i primi esperimenti di NMR a impulsi e si aprirono nuove linee di ricerca riguardanti i materiali ferroelettrici, i materiali magnetici, le transizioni di fase ed i solidi in generale, a cui hanno dato un notevole impulso Rigamonti e Borsa. In generale, la transizione all'utilizzo degli impulsi RF ha permesso un ampliamento importante degli ambiti di applicabilità della tecnica a livello mondiale, portando alla produzione commerciale di spettrometri orientati, ad esempio, alla NMR ad alta risoluzione, molto utilizzata in Chimica per finalità di caratterizzazione di composti molecolari ed in Biologia per studi sulle proteine, oppure alla Risonanza Magnetica per Immagini (MRI), che costituisce il principale sviluppo della NMR in ambito biomedico. 
L'attività del gruppo di Pavia ha avuto un notevole sviluppo a partire dagli anni ' 80 per quanto riguarda le attività nell'ambito della Fisica della Materia e della Fisica Applicata al settore Biomedico. Nella Fisica della Materia le principali indagini hanno riguardato i superconduttori, i magneti a bassa dimensione, il magnetismo frustrato, i magneti molecolari, i sistemi a elettroni fortemente correlati e, più recentemente, i rotori molecolari. Invece, nella Fisica Applicata al settore Biomedico sono stati condotti numerosi studi sui mezzi di contrasto per MRI, sulle nanoparticelle magnetiche per la teranostica e, nell'ultimo decennio, sulla polarizzazione dinamica dei nuclei. Dal 2019 è stato anche acquisito un tomografo per MRI destinato primariamente alla ricerca preclinica. Nei prossimi capitoli, verranno riportati alcuni esempi significativi di risultati ottenuti a Pavia, preceduti da un'introduzione generale alla problematica scientifica.

\subsection{La NMR ad impulsi ed i tempi di rilassamento degli spin nucleari}

L'acquisizione di NMR ad impulsi più semplice può essere effettuata applicando un campo magnetico statico $\vec{H}$ ad un campione attorno a cui viene avvolta una bobina in grado di applicare un campo magnetico $\vec{H}_{1}$ a Radiofrequenza. $\vec{H}_{1}$ deve essere contenuto nel piano normale al campo magnetico (Fig. 2).

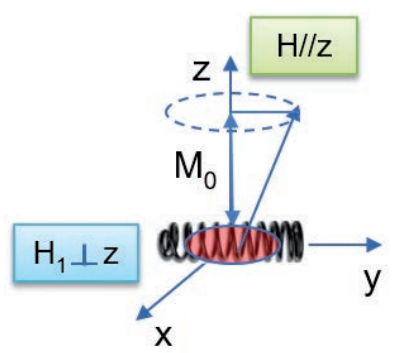

Fig. 2. Configurazione dell'esperimento NMR ad impulsi e magnetizzazione d'equilibrio.

All'inizio dell'esperimento gli spin dei nuclei del campione si allineano con una componente preferenziale parallela alla direzione del campo, $z$, e si genera la cosiddetta magnetizzazione nucleare $\vec{M}$, raffigurabile come un vettore. Essa precede attorno al campo $\vec{H}$ alla frequenza di Larmor $\omega_{L}=\gamma H$, dove $\gamma$ è il rapporto giromagnetico nucleare in $\mathrm{rad} /\left(\mathrm{s} \cdot\right.$ Tesla), mantenendo costante la componente $M_{z}=M_{0}$, a sua volta dipendente dalla densità di spin per unità di volume, dall'intensità di $\vec{H}$ 
e dalla temperatura (si veda per approfondimenti il paragrafo 3.2).

La condizione di risonanza magnetica si realizza applicando l'ulteriore campo $\vec{H}_{1}$ avente una componente rotante proprio alla pulsazione $\omega_{L}$. Poichè $\vec{M}$ ed $\vec{H}_{1}$ sono a riposo l'uno rispetto all'altro in un sistema di riferimento rotante a $\omega_{L}, \vec{H}_{1}$ è in grado di fare precedere $\vec{M}$ efficientemente attorno a sé nonostante esso sia normalmente molto più debole di $\vec{H}$. Se si applica $\vec{H}_{1}$ per un tempo limitato a piegare $\vec{M}$ nel piano $x y$, si parla di applicazione di impulso di $90^{\circ}$ (Fig. 3).
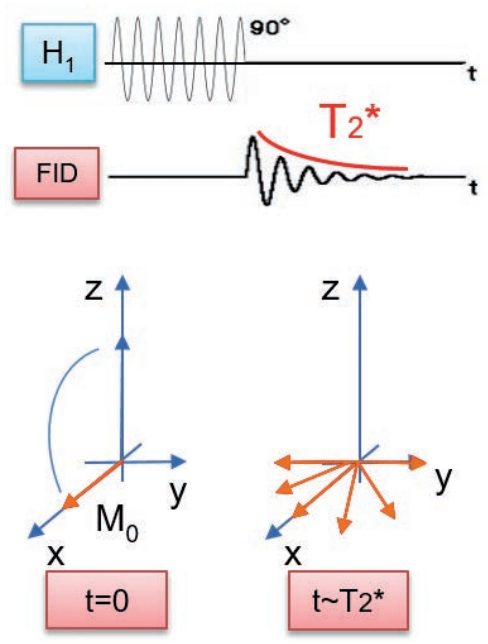

Fig. 3. Schema dell'esperimento di FID (in alto)

e defasamento progressivo degli spin al passare del tempo (in basso).

Dopo l'eccitazione, $\vec{M}$ tende a tornare all'equilibrio, precedendo attorno ad $\vec{H}$. Così facendo produce a sua volta un campo magnetico rotante a $\omega_{L}$ che viene detettato dalla stessa bobina nel piano $x y$ generando un potenziale elettrico oscillante a $\omega_{L}$. Tale segnale, detto di Free Induction Decay (FID), ha un'ampiezza proporzionale alla $M_{x y}$ a $\mathrm{t}=0$ che lo genera e decade esponenzialmente con un tempo tipico chiamato $T_{2}^{*}$, in una scala di tempo molto breve. Se l'impulso riesce a piegare efficacemente tutta la magnetizzazione nel piano $x y$, al termine dell'impulso $M_{x y}=M_{0}$. Il successivo decadimento origina dal defasamento reciproco dei segnali individuali degli spin del campione, $i$ quali sono soggetti a $\omega_{L}$ diverse tra loro, determinate dalla somma dei campi microscopici locali e dalle disomogeneità di $\vec{H}$ (Fig. 3). 
Per annullare l'effetto delle disomogeneità è necessario applicare l'eco di spin (Fig. 4). A seguito dell'impulso di $90^{\circ}$, il defasamento della FID che si verifica nel tempo $\tau$, normalmente superiore al $T_{2}^{*}$, viene recuperato applicando un ulteriore impulso a radiofrequenza di $180^{\circ}$. Quindi, dopo un ulteriore tempo di attesa pari di nuovo a $\tau$ e complessivamente al tempo di eco $T_{E}=2 \tau$, gli spin tornano alla fase iniziale ed il segnale somma si ricostruisce. All'aumento di $T_{E}$, l'intensità dell'eco decade comunque per effetto delle interazioni magnetiche locali irreversibili tra spin, questa volta, però, con il tempo di rilassamento spin-spin $T_{2}$, caratteristico del sistema nucleare e normalmente più lungo del $T_{2}{ }^{*}$.

L'intensità dell'eco è determinata primariamente da $T_{2}$, ma anche dal tempo di rilassamento spin-reticolo $T_{1}$, determinato dagli scambi energetici consentiti tra gli spin nucleari e le altre riserve energetiche del loro ambiente microscopico (elettroni, fononi, moti molecolari...). Il rilassamento spin-reticolo avviene su una scala di tempi in genere più lunga e determina il fenomeno di ritorno della magnetizzazione al proprio equilibrio termico lungo $z$. Ripetendo la sequenza spin echo ed accumulando il segnale come necessariamente avviene nella pratica per aumentare il rapporto segnale-rumore dell'esperimento, se il tempo di ripetizione $T_{R}$ della sequenza è sensibilmente più corto di $T_{1}$, ogni nuova eccitazione con l'impulso di $90^{\circ}$ potrà piegare nel piano $x y$ solo una porzione di $M_{0}$ e pertanto l'ampiezza iniziale dell'eco risulterà ridotta anche di un fattore dipendente da $T_{1}$. Complessivamente, per nuclei a spin $I=1 / 2$ come il protone, l'intensità dell'eco rispetta quindi la seguente dipendenza:

$$
E \propto M_{0} e^{-\frac{T_{E}}{T_{2}}}\left(1-e^{-\frac{T_{R}}{T_{1}}}\right)
$$

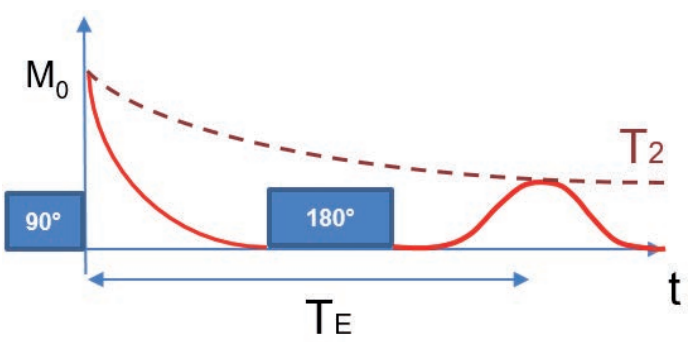

Fig. 4. Formazione dell'eco di spin. Per semplicità è riportato solo l'inviluppo rosso del segnale di FID visibile anche in Fig. 3. 
La determinazione di $T_{1}$ e $T_{2}$ è molto importante per caratterizzare i fenomeni locali rilevati dagli spin nucleari e comprenderne le proprietà. La NMR, inoltre, è anche una potente tecnica spettroscopica. Dalla trasformata di Fourier della FID è possibile infatti ottenere lo spettro o riga NMR, ricca di informazioni riguardanti le interazioni magnetiche e/o quadrupolari statiche e dinamiche a cui sono sottoposti i nuclei. Per maggiori approfondimenti sulla NMR si rimanda a testi specializzati del settore, ad esempio Fukushima 1981 e Slichter 1990.

\section{NMR NELLA MATERIA CONDENSATA: MAGNETI MOLECOLARI E SUPERCONDUTTORI}

\subsection{NMR in nanomagneti molecolari}

I magneti molecolari (MM) sono composti di dimensioni nanometriche contenenti un numero finito di centri paramagnetici collegati da ponti organici e racchiusi da una shell idrofobica non magnetica. La famiglia dei MM è ad oggi molto vasta e comprende aggregati di dimensioni variabili da alcuni angstrom ad alcuni nanometri con le più svariate proprietà e geometrie (Gatteschi 2011). Tra i più studiati vi sono stati storicamente i MM di metalli di transizione, quali ad esempio $\mathrm{Mn}_{12}$ (Fig. 5) e $\mathrm{Fe}_{8}$ (sono riportate le nomenclature brevi), sintetizzati a partire dagli anni '80 (Lis 1980) o, ad esempio, gli anelli di spin. In questi sistemi i momenti magnetici elettronici sono determinati dai soli momenti angolari di spin degli atomi, accoppiati dalle interazioni di superscambio $J_{i j} S_{i} S_{j}$ ferromagnetiche o antierromagnetiche interne alla molecola. Viceversa, le interazioni intermolecolari dipolari sono assai inferiori. Per temperature $(T)$ tali per cui l'energia termica supera le costanti di superscambio, $K_{B} T » J$, gli spin elettronici dei MM si comportano come in un sistema paramagnetico normale. Nel caso opposto, viceversa, gli spin elettronici sono vincolati in uno stato collettivo con spin totale elevato $S$ i cui livelli energetici, grazie alla taglia finita del sistema, sono quantizzati e discreti. Ogni MM si comporta pertanto come un nanomagnete con spin $S$ indipendente dai suoi vicini, tanto che si parla anche di magneti di singola molecola (single molecule magnets, SMM).

Lo studio dei SMM offre la possibilità unica di osservare effetti difficilmente osservabili nel continuo tridimensionale della materia condensata. Abbassando T, la molecola viene man mano intrappolata nello stato di spin fondamentale ed il tempo caratteristico in cui essa vi permane si 
allunga sensibilmente. Oltre all'interazione di scambio tra gli spin dei diversi centri magnetici, è presente, infatti, una forte anisotropia magnetica che tende ad indurre un allineamento preferenziale dello spin totale lungo un determinato asse o in un piano. In molti sistemi l'anisotropia ha un asse facile $z$ e l'interazione ad esso associata, di carattere perturbativo rispetto a quelle di superscambio, si può descrivere con un termine $D S_{z}^{2}$. Ogni stato S, quindi, si apre in tanti sottolivelli con separazione, presente anche in assenza di campo magnetico $(H)$ applicato, determinati da $D m_{s}^{2}$, dove $m_{s}$ è il numero quantico magnetico di spin variabile da $-S$ a $+S$. I livelli energetici sono pertanto sostanzialmente degeneri nel segno +/- e collocati in modo simmetrico rispetto alla barriera di potenziale che, se espressa in funzione dell'angolo tra l'asse facile e la magnetizzazione del SMM, risulta doppia, come si può vedere in Fig. 5. Quando il SMM è nel livello energetico più basso dello stato $S$ fondamentale, esso si comporta come un superparamagnete con un momento magnetico bistabile che può essere orientato parallelamente $\mathrm{o}$ antiparallelamente rispetto $\mathrm{a} z$.

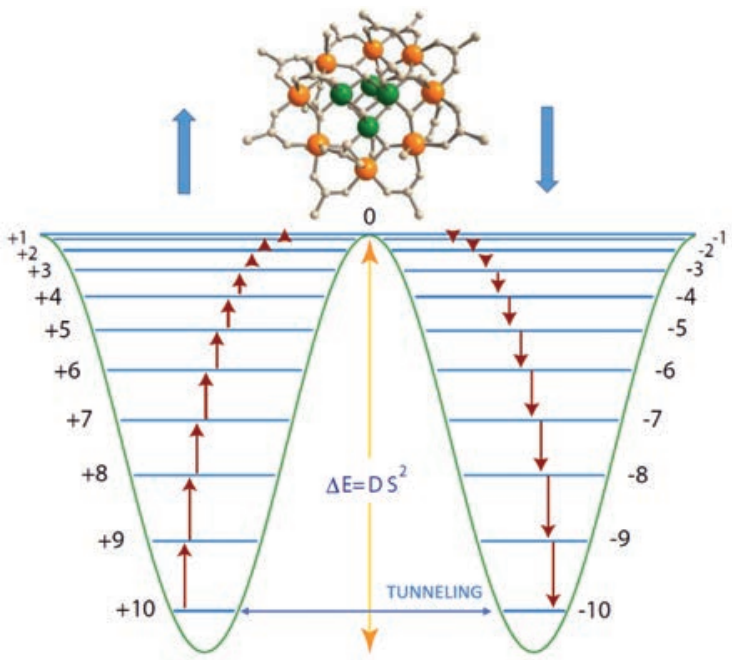

Fig. 5. Raffigurazione della molecola $M n_{12}$ e struttura dei livelli energetici del multipletto fondamentale $S=10$ determinata dall'anisotropia magnetica DSz. Sono segnalate con le frecce le possibili transizioni dovute all' eccitazione termica ed al tunneling nello stato fondamentale.

Questa proprietà ha presto stimolato un'intensa ricerca finalizzata ad utilizzare tali stati di spin, ad esempio come bit logici magnetici per nanomemorie ad altissima densità (Sessoli 1993), come qubit nella 
Quantum Information (Leuenberger 2001, Timco 2009), in dispositivi spintronici (Bogani 2008). In vista delle suddette applicazioni, chiaramente, il "tempo di vita" del bit o del qubit devono divenire appropriatamente lunghi e le operazioni di scrittura, manipolazione e lettura accessibili a $T$ di lavoro non eccessivamente basse (almeno a $78 \mathrm{~K}, T$ di ebollizione dell'azoto liquido) e senza l'interferenza di fenomeni spuri. Ciò può essere valutato misurando sperimentalmente il tempo di rilassamento della magnetizzazione del SMM.

Per $K_{B} T \cong D S^{2}$, tutti i sottostati $m_{s}$ sono popolati secondo la statistica di Boltzmann e la magnetizzazione del SMM può ruotare per mezzo delle eccitazioni termiche. $\tau$ ha quindi un comportamento attivato $e^{\Delta E / K_{B} T}$, con la barriera energetica $\Delta E=D S^{2}$. Quando $K_{B} T \ll D S^{2}$, al di sotto di una $T$ di bloccaggio, il SMM è congelato. In questo regime $\tau$ è determinato anche da fenomeni di decoerenza quantistica. Infatti, la doppia degenerazione dei livelli di spin a cavallo della barriera di anisotropia magnetica crea, ad esempio, una condizione favorevole al verificarsi del Tunneling quantistico della Magnetizzazione, dove lo spin della molecola si trova a transire tra due possibili stati $+/-m_{s}$.

Sfortunatamente nei SMM di metalli di transizione i fenomeni appena descritti si verificano generalmente a $T$ molto basse: la barriera di anisotropia del $\mathrm{Mn}_{12}$ è dell'ordine di $66 \mathrm{~K}$, mentre la molecola si blocca solo a pochi gradi Kelvin con un $\tau$ che diventa dell'ordine dei giorni. Per questo motivo, più recentemente, è stata dedicata molta attenzione anche a complessi contenenti ioni di terre rare. In questa famiglia (Dey 2018) i cosiddetti double-decker, in cui due molecole organiche quali ftalocianine o porfirazine racchiudono un solo atomo di Terbio, Disprosio, Gadolinio o altri, chiamati anche Single Ion Magnets (SIM), sono stati i più studiati. Nei SIM di terre rare, gli stati energetici sono determinati dal momento angolare totale $J=L+S$. Tra i primi composti analizzati dal punto di vista magnetico, storicamente il $\left[\mathrm{TbPc}_{2}\right][\mathrm{TBA}]$ è subito apparso molto attraente per ipotetici risvolti tecnologici poiché la separazione tra i primi sottolivelli $m_{J}= \pm 6$ e $m_{J}=$ \pm 5 del multipletto fondamentale $J=6$, indotta dall'anisotropia magnetica, era stata stimata inizialmente attorno a $628 \mathrm{~K}$ (Ishikawa 2003), almeno un ordine di grandezza maggiore di quella rinvenuta nel $\mathrm{Mn}_{12}$-Ac. Molto recentemente i complessi di $\mathrm{TbPc}_{2}$ sono stati identificati addirittura come possibili qudit per lo sviluppo di dispositivi molecolari in grado di eseguire algoritmi quantistici (Moreno-Pineda 2018). Nuovi composti di Disprosio, invece, hanno dimostrato una bistabilità magnetica ed un comportamento isteretico a $T$ record di circa $60 \mathrm{~K}$, poco infe- 
riore a quella dell'azoto liquido, diventando molecole di punta per lo stoccaggio magnetico di informazione (Sessoli 2017).

Come richiamato qui sopra, la bistabilità dello stato fondamentale rende i MM utilizzabili come bit classici in cui sono presenti solo due stati logici, corrispondenti a bit $|0>0| 1\rangle$, tuttavia l'anisotropia magnetica e/o l'applicazione di campi magnetici esterni possono modificare lo stato fondamentale e farlo diventare una opportuna sovrapposizione di questi due stati logici $a|0>+b| 1>$, ovvero un bit quantistico o qubit che permette lo stoccaggio su un numero elevatissimo di stati al variare di $a$ e $b$. La possibilità di utilizzare queste molecole come unità logiche dipende dal tempo di decoerenza quantistica $\tau$, tempo caratteristico su cui si preserva un certo stato di spin. Poiché le fluttuazioni di spin danno luogo a fluttuazioni nel campo iperfine sondato dai nuclei della molecola, il tempo di decoerenza può essere determinato attraverso misure del tempo di rilassamento spin-reticolo $T_{1}$ (Borsa 2006, Borsa 2007). In un tipico $\mathrm{MM}$ come il $\mathrm{TbPc}_{2}$ neutro si osserva un comportamento dell' $1 / T_{1}$ caratterizzato da un massimo a $T$ per le quali il tempo di decoerenza è dell'ordine della frequenza di Larmor nucleare (circa $100 \mathrm{MHz}$ ) e una diminuzione a più bassa T (Branzoli 2009).
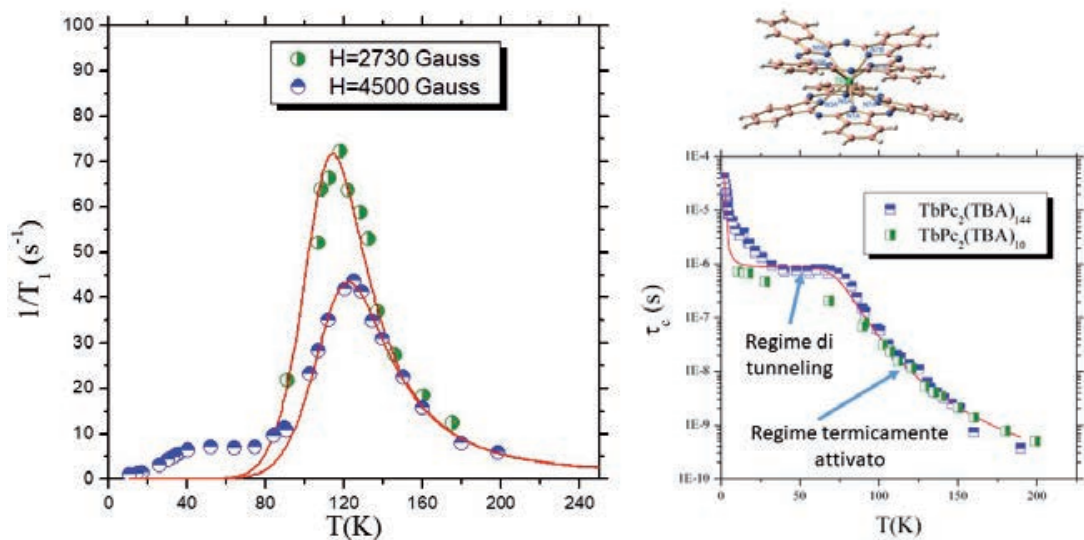

Fig. 6. (sinistra) Andamento con la $\mathrm{T}$ dell' $1 / T_{1}$ dei nuclei di ${ }^{1} \mathrm{H}$ in magneti molecolari di $\mathrm{TbPC}_{2}(\mathrm{TBA})_{144}$ (in alto a destra è raffigurato il $\left.\mathrm{TbP}_{2}\right)$ a due $\mathrm{H}$ differenti. Le curve a tratto continuo mostrano l'andamento teorico previsto per l' $1 / T_{1}$ assumendo un comportamento termicamente attivato delle fluttuazioni di spin. È evidente il cambio di regime sotto gli $80 \mathrm{~K}$ verso un regime di tunneling. (destra) Andamento con la $T$ del tempo di decoerenza con evidenziati i due regimi.

Le misure di $T_{1}$ permettono non solo di determinare l'andamento funzionale di $\tau$ ma anche di quantificarlo. Come illustrato in Fig. 6, si 
osservano due regimi: uno a più alta $T$, caratterizzato da fluttuazioni fra lo stato fondamentale e i primi stati eccitati, e uno a più bassa $T$ nel quale $\tau$ è pressoché indipendente dalla $T$ e le fluttuazioni avvengono all'interno dello stato fondamentale bistabile. Il gruppo di Pavia ha dato contributi significativi in questo settore dagli anni '90 ad oggi.

\subsection{NMR in superconduttori ad alta Temperatura}

La scoperta della superconduttività ad alta temperatura $(T)$ nei cuprati da parte di Bednorz e Muller nel 1986 (Bednorz 1986) ha portato a una rivoluzione epocale nella Fisica della Materia e nelle applicazioni dei materiali superconduttori. Difatti il raggiungimento di temperature di transizione superiori alla $T$ di ebollizione dell'azoto liquido $(78 \mathrm{~K})$ permette l'applicazione dei superconduttori al settore biomedicale, dello stoccaggio dell'energia o nelle tecnologie dell'informazione a costi assai più contenuti. I superconduttori ad alta temperatura $\left(\mathrm{HT}_{c} \mathrm{~S}\right)$ a base di Rame e a partire dal 2009 anche quelli a base di Ferro, hanno avuto una notevole rilevanza anche per quanto riguarda studi di natura fondamentale e ad oggi, 35 anni dopo la loro scoperta, il meccanismo che porta alla superconduttività ad alta $T$ non è stato del tutto chiarito. La ragione è che in questi materiali, al contrario dei metalli convenzionali, le scale di energia caratteristiche delle varie eccitazioni, di carica, di spin, orbitali e vibrazionali non sono ben separate e le forti correlazioni elettroniche e la competizione fra queste scale di energia rende i loro diagrammi di fase particolarmente complessi e interessanti. Difatti vi è probabilmente tuttora un maggiore interesse nello studio degli aspetti di base dei cuprati e dei superconduttori a base di Ferro rispetto ai superconduttori convenzionali a $T$ ambiente (ma a pressioni di $200 \mathrm{GPa}$ ) scoperti nel 2020 (Sneider 2020). L'applicazione dei $\mathrm{HT}_{\mathrm{c}} \mathrm{S}$ può essere tuttavia limitata in presenza di campi magnetici. Questi materiali appartengono alla famiglia dei superconduttori di tipo II, caratterizzati da una parziale penetrazione del campo magnetico $(H)$ al loro interno (Fig. 7) per $H$ di intensità di qualche decina di $\mathrm{mT}$.

$H$ penetra sotto forma di linee dette flussoni poiché il flusso di campo magnetico attraverso la superficie perpendicolare alla singola linea di flusso ha un valore ben preciso pari a un quanto di flusso $\Phi_{0}=2,0678 \times 10^{-15} \mathrm{~T} \cdot \mathrm{m}^{2}$ (Rigamonti 2015). In presenza di una corrente elettrica oppure a causa dell'attivazione termica le linee di flusso tendono a muoversi e a dare luogo a dinamiche dissipative, facendo sì che, nonostante il materiale sia superconduttore, la resistenza elettrica diventi non nulla. Per ovviare a questo inconveniente è necessario anco- 
rare le linee di flusso, utilizzando impurezze, ad esempio. L'efficacia di questo ancoraggio dipende da caratteristiche intrinseche del materiale (lunghezza di coerenza, anisotropia, etc...) e da parametri estrinseci (tipo di impurezza, $H$, T, etc...). E' pertanto di notevole importanza poter studiare queste dinamiche e come varia la distribuzione di campo magnetico indotta dai flussoni all'interno del superconduttore.

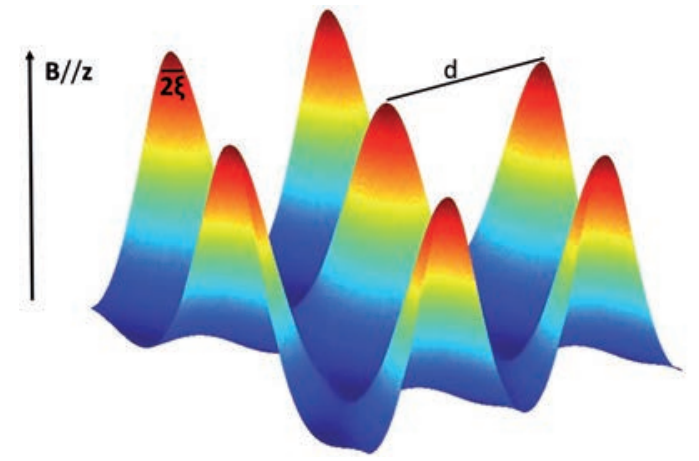

Fig. 7. Rappresentazione dell'intensità del campo magnetico (rosso intensità massima, blu minima) nel reticolo di linee di flusso di un superconduttore di tipo II. d è il passo reticolare, dell'ordine del centinaio di Angstrom per campi magnetici dell'ordine del Tesla, e $\xi$ la lunghezza di coerenza che determina il raggio della linea di flusso. Il flusso del campo magnetico attraverso una cella primitiva è quantizzato e pari a $\Phi_{0}$.

I nuclei costituiscono una rete nanoscopica di sensori di campo magnetico che permette, grazie all'NMR, di mappare finemente la distribuzione di campi magnetici all'interno del superconduttore. Gli stessi nuclei sondano le fluttuazioni di campo magnetico indotte dalle dinamiche dei flussoni e quindi è possibile determinare i tempi caratteristici con cui si spostano le linee di campo magnetico al variare di diversi parametri, come $H$ e $T$, partendo da misure di $T_{1}$ in NMR (Rigamonti 1998).

Questi studi hanno permesso, sia nei cuprati che nei superconduttori a base di Ferro, di evidenziare diverse fasi formate dal reticolo di linee di flusso: fasi liquide con moti fortemente dissipativi, per valori elevati di intensità di $H$ e $T$, e una fase solida o vetrosa, a seconda della concentrazione di difetti, per bassi valori di campo $H$ e $T$ (Corti 1996). Abbassando ulteriormente $H$, il reticolo di flussoni scompare e il campo magnetico viene espulso completamente dall'interno del superconduttore. In Fig. 8 si vede che quando le dinamiche dei flussoni, per un dato valore di $H$ e $T$, raggiungono frequenze caratteristiche dell'ordine della frequenza di Larmor nucleare si osserva un massimo in $1 / T_{1}$ 
(Corti 1996, Rigamonti 1998, Carretta 2020). La dipendenza del tempo di correlazione stimato da queste misure dalla $T$ può avere un comportamento termicamente attivato, indipendente dalla $T$ o seguire la legge di Vogel-Fulcher caratteristica delle transizioni vetrose, come osservato nei superconduttori di $\mathrm{Ba}\left(\mathrm{Fe}_{1-\mathrm{x}} \mathrm{Rh}_{\mathrm{x}}\right)_{2} \mathrm{As}_{2}$ (Bossoni 2013) (Fig. 9).
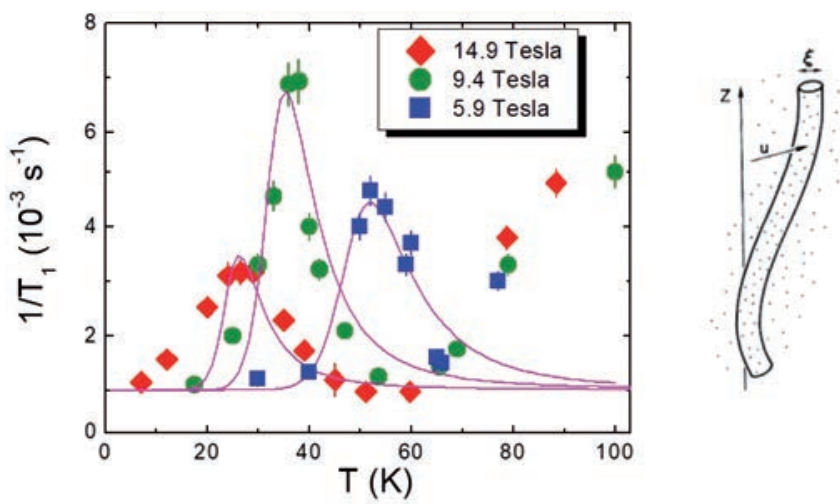

Fig. 8. Andamento con la $\mathrm{T}$ dell' $1 / \mathrm{T}_{1}$ dei nuclei di ${ }^{89} \mathrm{Y}$ in superconduttori di $\mathrm{YBa}_{2} \mathrm{Cu}_{4} \mathrm{O}_{8}$ (temperatura critica $T_{c}=80 \mathrm{~K}$ in campo nullo) per tre valori diversi di $\mathrm{H}$ (Corti 1996). Il picco osservato indica che per un determinato valore di $\mathrm{H}$ le fluttuazioni delle linee di flusso, schematizzate sulla destra per $H$ diretto lungo $z$, sono caratterizzate da frequenze dell'ordine della frequenza di Larmor.

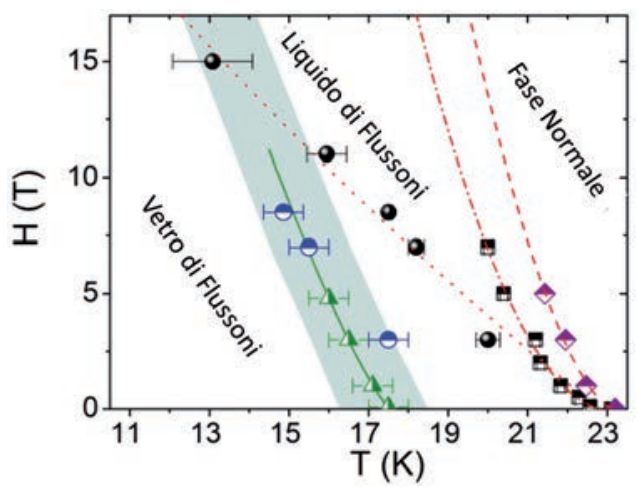

Fig. 9. Diagramma di fase campo magnetico (H)-temperatura (T) per il superconduttore $\mathrm{Ba}\left(\mathrm{Fe}_{0.93} \mathrm{R} \mathrm{b}_{0.07}\right)_{2} A s_{2}$ derivato a partire da misure di $1 / T_{1}$ su nuclei di ${ }^{75} A s$ e da misure di suscettività magnetica (Bossoni 2013). Viene evidenziata una fase a bassa T di vetro di flussoni separata da una fase liquida. I cerchi neri evidenziano la $T$ di irreversibilità alla quale la frequenza di fluttuazione dei flussoni raggiunge la frequenza caratteristica di misura e pertanto non è univocamente definita. I quadrati e $i$ rombi indicano la $T$ di transizione verso la fase normale per $H$ paralleli al piano ab o perpendicolari ad esso, rispettivamente. 


\section{APPLICAZIONI BIOMEDICHE: MRI, DNP ED IL NUOVO CENTRO PRECLINICO}

\subsection{La Tomografia a Risonanza Magnetica Nucleare (MRI) e gli agenti di contrasto}

Una delle applicazioni più rilevanti della NMR avviene in campo biomedico con la tecnica denominata Magnetic Resonance Imaging (MRI) che permette di acquisire immagini di specifiche sezioni di un campione, fornendo una raffigurazione dei dettagli interni con ottima risoluzione spaziale (da cui l'utilizzo del termine tomografia). La MRI è diffusamente effettuata in campo clinico per fini diagnostici, normalmente con campi magnetici dell'ordine di 1,5 o 3 Tesla, ma ha anche un ruolo cruciale nella ricerca scientifica, sia in studi su pazienti che in studi di modelli animali, a campi magnetici ancora più elevati (Ladd 2018). Nel caso di studi in vivo, in particolare, la MRI è particolarmente indicata per l'investigazione di tessuti molli ed organi interni perché può ricevere direttamente il segnale proveniente dagli spin nucleari dell'idrogeno (protone) delle molecole d'acqua o grasso, evitando in molti casi l'asportazione o il prelievo per ulteriori analisi esterne. Inoltre, sia in ambito clinico che nella ricerca, essa è uno strumento di esame molto prezioso per la sua non invasività. Infatti, la MRI utilizza radiazioni non ionizzanti e può essere pertanto ripetuta anche in studi prolungati a tempi ravvicinati, senza indurre danni al paziente.

Un modello di tomografo di ultima generazione per studi preclinici avanzati di MRI risiede presso il Laboratorio di Imaging a Risonanza Magnetica e Micro Tomografia a raggi X del Centro Grandi Strumenti dell'Università degli Studi di Pavia (Fig. 10). Esso è dotato di un magnete superconduttore con bore orizzontale che produce un campo magnetico statico $(H)$ da 7 Tesla, sufficientemente alto per risolvere dettagli strutturali submillimetrici. In un esperimento tipico, il campione oggetto di studio viene posto all'interno del magnete e, come nella NMR, è sottoposto a sequenze di acquisizione e ricezione basate su impulsi a radiofrequenza $(\mathrm{RF})$, accordati alla frequenza di Larmor tipica del nucleo sotto esame, $v_{\mathrm{L}}=\omega_{\mathrm{L}} / 2 \pi$ pari a circa $300 \mathrm{MHz}$ a 7 Tesla nel caso del protone (si veda il paragrafo 1.2 per richiami sulla NMR).

Per ricavare un'immagine nell'esperimento MRI è necessario rendere distinguibili i segnali provenienti dai protoni collocati in diverse regioni del campione. Si procede quindi anche con l'applicazione dei gra- 
dienti di campo magnetico $(G)$, prodotti da bobine supplementari installate all'interno del magnete: in questo modo, ad esempio, nuclei collocati in una posizione $\mathrm{x}_{0}$ lungo la direzione $x$ del gradiente lineare $G_{x}$ producono segnali con pulsazione caratteristica $\omega=\omega_{L}+\gamma G_{x} x_{0}$. Proprio questa informazione, quindi, viene utilizzata per assegnare a ritroso a tali nuclei la posizione $x_{0}$ nello spazio nel processo di ricostruzione dell'immagine. Primariamente i gradienti vengono combinati ad impulsi RF selettivi nella fase di slice selection che permette di ottenere le immagini delle cosiddette "fette" rappresentative del campione, tipiche della tomografia. Essi vengono inoltre applicati per codificare in frequenza ed in fase $i$ segnali dei nuclei ed identificarne pertanto la posizione in 2 o 3 dimensioni, per ricostruire immagini e volumi (Berstein 2004).

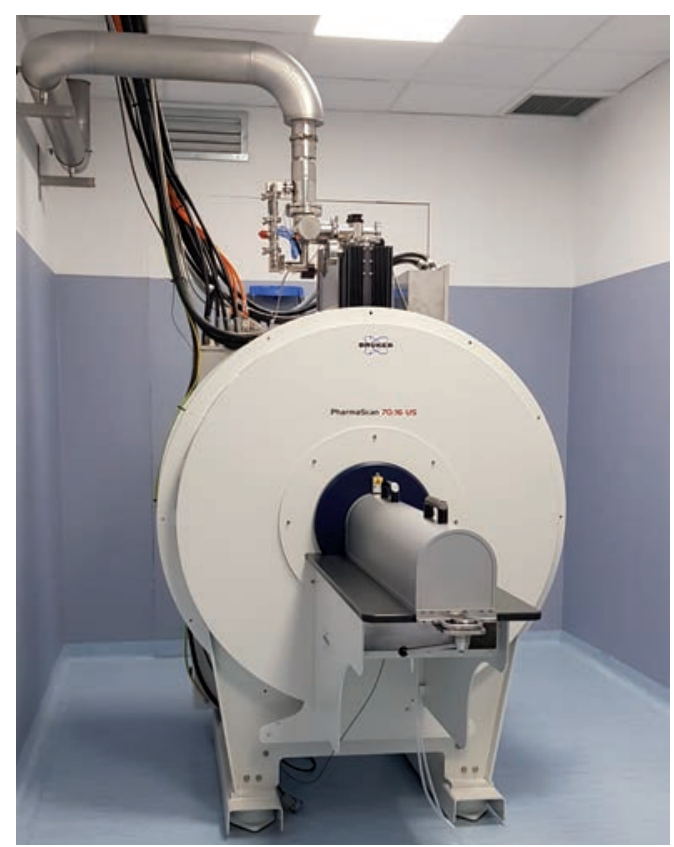

Fig. 10. Tomografo MRI per ricerca preclinica dell'Università degli Studi di Pavia.

La grandezza minima risolvibile, ovvero la scala spaziale minima dei fenomeni microscopici analizzabili all'interno di un'immagine MRI, dipende molto dalla strumentazione utilizzata e dal protocollo applicato. La risoluzione tipica delle immagini per studi morfologici clinici nei pazienti è dell'ordine del millimetro mentre nei tomografi 
destinati alla ricerca può scendere a poche centinaia di micron nell'uomo (Lüsebrink 2017) e anche sotto al centinaio di micron nei modelli animali (Pautler 2004).

Il segnale MRI è generato, come già detto, dalla presenza di protoni. Tuttavia ogni voxel di una specifica regione, un tessuto od un liquido biologico è contraddistinto nell'immagine MRI da un'intensità in scala di grigi caratteristica, data dalle condizioni e dai parametri sperimentali $\left(H\right.$, temperatura $T$, tempo di echo $T_{E}$, tempo di ripetizione $T_{R} \ldots$ ) e dalle proprietà fisiche microscopiche locali (spin density $\rho$, tempo di rilassamento spin-reticolo $T_{1}$, tempo di rilassamento spin-spin $T_{2}$ ). La MRI è una tecnica diagnostica molto efficace proprio perché in molte patologie $\rho, T_{1}$ e $T_{2}$ subiscono delle variazioni anomale e quindi i tessuti malati sono caratterizzati da un contrasto visibilmente modificato rispetto ai tessuti sani. Inoltre, vari tessuti o liquidi biologici possono avere tempi di rilassamento molto diversi tra loro anche in un organismo sano: ad esempio, a 7 Tesla nel cervello il $T_{1}$ dell'acqua e dei tessuti è di alcuni secondi (Deruelle 2020) mentre il $T_{1}$ del grasso è dell'ordine delle centinaia di ms.

Il contrasto tra diversi tessuti può essere quindi variato regolando opportunamente i parametri di acquisizione, in modo da acquisire un'immagine cosiddetta 'pesata' nella densità protonica (la $\rho$ dei protoni), pesata in $T_{1}$ o pesata in $T_{2}$. All'occorrenza è possibile aumentare o diminuire, ad esempio, i segnali dei liquidi o dei grassi. In Fig. 11 si mostrano ad esempio due immagini della regione del cervelletto e del tronco encefalico nel cervello di un ratto sano, acquisite nel Centro di Imaging Preclinico di Pavia con sequenze basate sull'eco di spin (paragrafo 1.2, formula 1.1): l'immagine di sinistra è pesata in $T_{2}$ (con $T_{E}$ dell'ordine o maggiore di $T_{2}$ e $T_{R} \gg>T_{1}$ ) e quella di destra pesata nella densità protonica (con $T_{E}<<T_{2}$ e $\left.T_{R}>>T_{1}\right)(\mathrm{M}$. Peviani, Univ. Pavia, in fase di pubblicazione). L'immagine pesata in $T_{2}$ permette di contrastare efficacemente i vasi (ipointensi) ed i fluidi (iperintensi). L'immagine pesata in densità protonica offre un buon contrasto tra materia grigia (di media intensità) e materia bianca (ipointensa). Il contrasto è opportunamente stabilito per studiare al meglio diverse regioni o patologie ed a seconda dell'obiettivo diagnostico o sperimentale. Ad esempio, per rilevare la presenza di edemi o ischemia (Ho 2012), sono utilizzabili le sequenze $T_{2}$-pesate, mentre per contrastare meglio i tessuti tumorali (Damadian 1971) od il tessuto adiposo (Marzola 2016) in alcuni casi si può applicare delle sequenze $T_{1}$-pesate. 

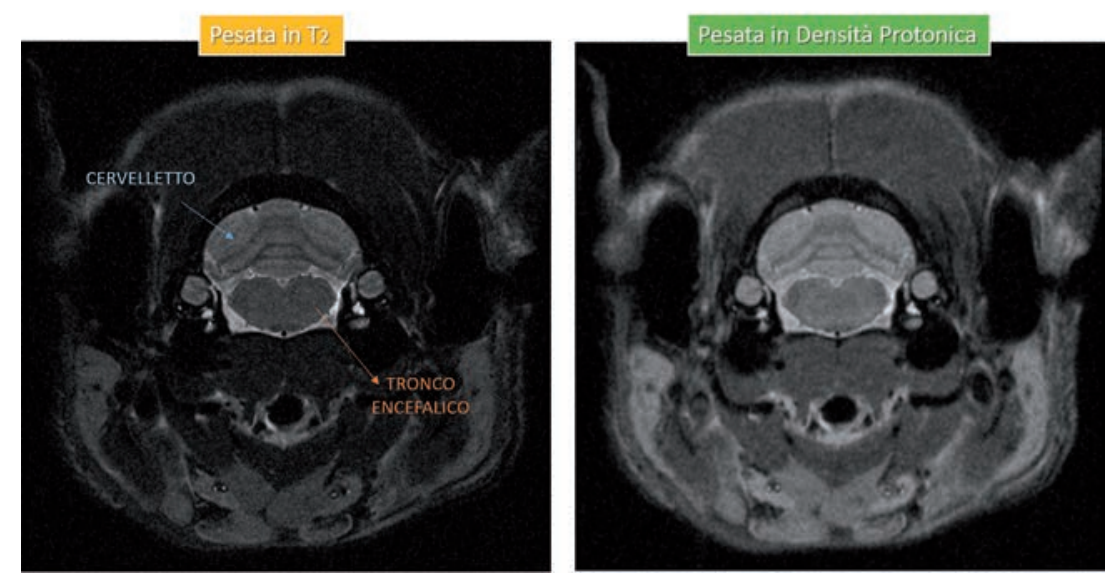

Fig. 11. Sezione coronale raffigurante il cervelletto ed il tronco encefalico di un ratto sano ottenuta in vivo con una sequenza basata sullo spin echo ed impostata con diversi parametri di acquisizione, in modo da determinare un peso in $T_{2}$ (a sinistra) o in proton density (a destra).

Oltre alle spin echo, vi sono anche sequenze in cui l'eco è ottenuto tramite la sola applicazione di gradienti (gradient echo) ed in cui il segnale MRI viene pertanto pesato in $T_{2}^{*}$ : esse possono mettere in evidenza eterogeneità ed interfacce nella composizione del campione ed, in genere, salti nella suscettività magnetica. Le sequenze basate sull'eco di gradiente sono più interessate da artefatti rispetto alle spin echo ma sono indispensabili per effettuare acquisizioni veloci ad esempio nelle applicazioni Cardio MRI sul cuore (Saeed 2015) e nell'angiografia (Hartung 2011), poiché il loro tempo di acquisizione è breve abbastanza da permettere una sincronizzazione con movimenti e flussi veloci.

In effetti, le sequenze basate sull'eco di spin richiedono normalmente tempi di acquisizione molto lunghi (potrebbero arrivare a diverse ore), pertanto è stata condotta un'intensa ricerca per realizzare una loro accelerazione. Le sequenze RARE (Henning 1986), ad esempio, permettono di acquisire nel $T_{R}$ più echi applicando dopo il primo impulso di $90^{\circ}$ un numero $N_{E}>1$ di impulsi di $180^{\circ}$, distanziati tra loro nel tempo di un intervallo $T_{E}$. Ad ogni eco è eseguibile uno step aggiuntivo della codifica di fase in modo da ridurre il tempo di acquisizione di un fattore $N_{E}$. Nello stesso $T_{R}$, inoltre si possono eccitare più fette, di conseguenza in un tempo sperimentale dell'ordine della decina di minuti diviene accessibile l'acquisizione di immagini in vivo da regioni 
anatomiche estese, come mostra l'esempio in Fig. 12, ed un'identificazione più veloce delle alterazioni in tessuti malati (M. Peviani, Univ. Pavia, in fase di pubblicazione).

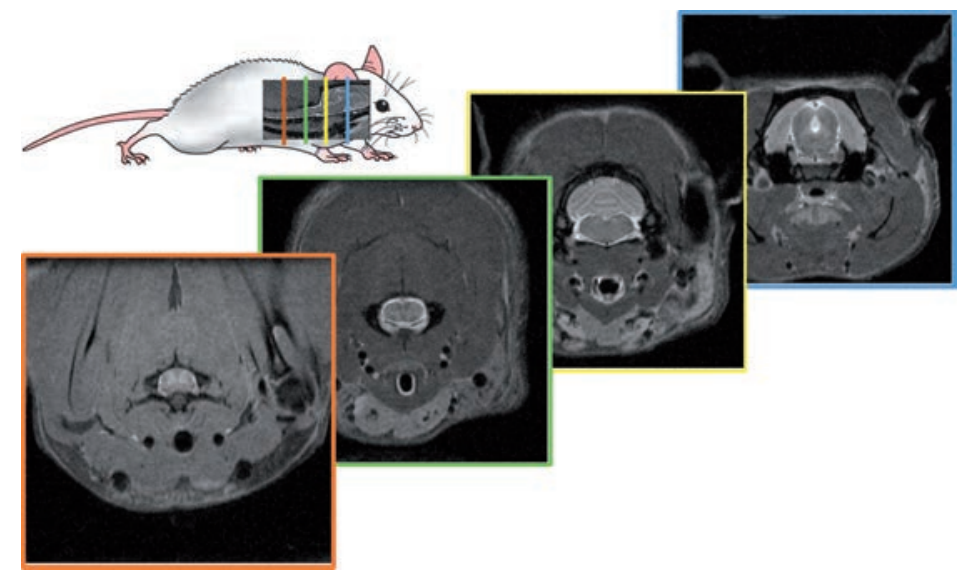

Fig. 12. Esempi di acquisizione multi slice in vivo con una sequenza RARE in un ratto sano.

Ancora più recentemente la disponibilità di potenze di computazione sempre più elevate ha permesso anche di generare strategie combinate per accelerare efficacemente gli esperimenti. L'MRI Fingerprinting ( $\mathrm{Ma} 2013$ ) infatti è un approccio innovativo basato su acquisizioni molto veloci che forniscono "un'impronta" del segnale. Questa è interpretata sulla base di un dizionario contenente un gran numero di simulazioni e con tecniche di pattern recognition vengono ricostruite simultaneamente vere e proprie mappe quantitative dei parametri MRI di tutti i pixel di un'immagine. Dalla sua ideazione l'MRI Fingerprinting è oggi in rapida espansione e molti studi riguardano la sua futura traslazione in ambito clinico (Mehta 2019).

Gli esperimenti MRI più avanzati si basano anche su altri importanti fenomeni microscopici e fisiologici, quali ad esempio la diffusione delle molecole di acqua, la perfusione e l'ossigenazione dei tessuti. La diffusione è alla base delle sequenze di Diffusion Weighted Imaging e più nello specifico delle sue implementazioni più all'avanguardia, come la Diffusion Tensor Imaging (Alexander 2007) e la trattografia (Yamada 2009). In queste sequenze il contrasto dipende dal movimento dell'acqua nello spazio ed è possibile pertanto studiare le proprietà di strutture anisotrope in cui essa procede lungo direzioni preferenziali, come ad esempio la materia bianca del cervello e del midollo spinale e le relative 
degenerazioni patologiche. La celebrata MRI funzionale, invece, permette con vari protocolli di distinguere le aree attivate nel cervello e nel midollo spinale sulla base del livello di ossigenazione del sangue (sequenza BOLD), la perfusione o la dipendenza locale della suscettività magnetica (Ogawa 1990, Mandino 2020). Tecniche recenti denominate CEST (Chemical Exchenge Saturation Transfer) MRI (Wu 2016) utilizzano invece il fenomeno dello scambio chimico tra i protoni dell'acqua e quelli di altri composti, rendendo possibile mappare la concentrazione di agenti e molecole contenute nei tessuti anche in concentrazioni molto piccole. Esse appartengono a pieno titolo alla classe delle tecniche di imaging molecolare e sono molto promettenti, ad esempio, per lo studio del microambiente tumorale (Prasad 2021).

Con il tomografo MRI sono acquisibili inoltre gli spettri NMR di una o più regioni localizzate nel corpo. Questa specifica applicazione è chiamata Magnetic Resonance Spectroscopy (MRS) ed è ottimale per ricavare la concentrazione dell'acqua e di altre molecole presenti dei tessuti. Tra i composti quantificabili simultaneamente e con accuratezza vi sono svariati metaboliti quali, ad esempio nel cervello, $\mathrm{N}$-acetil aspartato (NAA), colina (Cho), creatina (Cr), myo inositolo (mIns), composti del glutammato (Glu) e glutammina (Gln), Lattato (Lac) (Pfeuffer 1999, Duarte 2012), rilevanti per lo studio delle malattie neurodegenerative (Öz 2014), i tumori (Horskà 2010) e i disordini neurologici. La MRS è in grado di rivelare efficacemente quali metaboliti fungono da biomarkers predittivi, se essi subiscono modifiche sostanziali precoci (Filibian 2012).

Negli ultimi decenni si sono fortemente sviluppate a Pavia (Casula 2010, Orlando 2016, Basini 2019) anche alcune linee di ricerca riguardanti gli agenti di contrasto, composti in grado di alterare localmente i tempi di rilassamento dei tessuti patologici al fine di renderli maggiormente distinguibili all'interno dell'immagine MRI rispetto ai tessuti sani. In Fig. 13 si riporta un esempio dell'effetto di un agente di contrasto in vitro al variare della concentrazione.

Tra gli agenti di contrasto più utilizzati si trovano alcuni composti paramagnetici di Terre Rare (in particolare Gadolinio) detti anche "positivi", disponibili anche commercialmente e utilizzabili in ambito clinico, che rendono iperintense le zone da essi raggiunte nelle immagini pesate in $T_{1}$. Inoltre esistono vari tipi di composti superparamagnetici, come le nanoparticelle magnetiche a base di ossidi di Ferro, detti anche agenti di contrasto "negativi", i quali, viceversa, rendono ipointense le regioni da essi raggiunte nelle immagini pesate in $T_{2}$ (Xiao 
2016). Tra le applicazioni principali degli agenti di contrasto vi sono state per anni l'angiografia MRI (Hartung 2011, Herborn 2012), lo studio dei tumori, anche in organi specifici (Zhou 2013, Akhtar 2021), ed i relativi processi infiammatori (Serkova 2017).

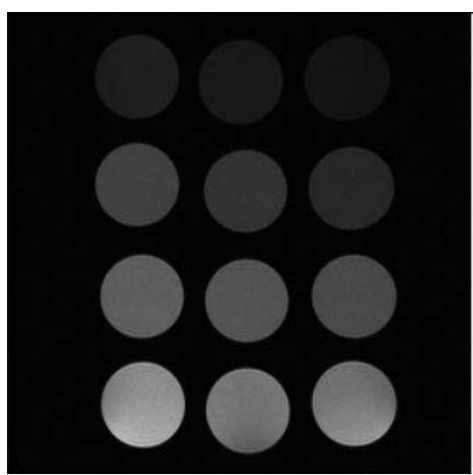

Fig. 13. Effetto della concentrazione di $\mathrm{MnCl}_{2}$ in soluzione acquosa. Al crescere della concentrazione (pari a $0.05 \mathrm{mM}$ nella prima fiala in basso a sinistra fino a $0.45 \mathrm{mM}$ nell'ultima fiala in alto a destra) l'agente di contrasto accorcia il $T_{2}$ e le soluzioni a maggior concentrazione divengono man mano ipointense in immagini $T_{2}$ pesate.

In particolare le nanoparticelle magnetiche sono tuttora considerate materiali altamente promettenti per lo sviluppo della teranostica (Jeong 2018). Esse sono composte essenzialmente di un core magnetico ed una shell organica biocompatibile funzionalizzabile per effettuare l'imaging multimodale (es. MRI e imaging ottico), l'ancoraggio di anticorpi nei tessuti in modo selettivo ed il rilascio locale di farmaci e/o di altri trattamenti, per esempio l'ipertermia magnetica. Le proprietà di questi composti, inclusa la tossicità, dipendono pesantemente dal core magnetico, dalla loro sintesi e dalla shell di copertura, dalla morfologia e dalla struttura e pertanto la caratterizzazione tramite tecniche basate sull'NMR ha una grande importanza per permettere l'ottimizzazione di tali composti in vista della somministrazione in vivo per la MRI. Tra gli ossidi di ferro, i più utilizzati per la sintesi sono la magnetite e la maghemite i quali inducono all'interno dei tessuti in cui vengono veicolati un accorciamento dei tempi di rilassamento, in genere più marcato per il $T_{2}$, e più precisamente un incremento della rilassività, intesa come l'inverso dei tempi di rilassamento per unità di concentrazione dell'agente di contrasto (Lascialfari 2013).

Nell'ottica di aumentare l'efficienza, studi molto recenti hanno 
messo in luce come nanoparticelle superparamagnetiche di maghemite ricoperte con polielettroliti possono raggiungere per alcuni diametri un rapporto $r_{2} / r_{1} 3$ volte maggiore degli agenti commerciali, lasciando intravedere verosimilmente una riduzione della dose con cui potrebbero essere somministrate in ambito clinico (Brero 2020).

Per finire, nonostante la MRI sia uno strumento ampiamente utilizzato, va tenuto presente che la durata delle acquisizioni e la limitata sensibilità verso volumi molto piccoli oppure composti presenti in basse concentrazioni o, infine, non contenenti idrogeno, la rendono in alcuni casi meno indicata di altre tecniche diagnostiche, quali ad esempio la PET per la sua alta sensibilità e specificità, soprattutto per la detezione di tumori di piccole dimensioni, o la CT per la sua risoluzione spaziale di norma più elevata. Nell'ultimo decennio, tuttavia, una modalità diagnostica particolarmente innovativa, nota come MRI iperpolarizzata, ha permesso di effettuare l'imaging di composti iniettati in piccola concentrazione nell'organismo e seguirne il metabolismo in tempo reale. All'Università di Pavia si studia in particolare l'applicazione della tecnica di iperpolarizzazione chiamata Polarizzazione Dinamica dei nuclei a molecole di interesse biologico, utili alla rilevazione di patologie con alterazioni al metabolismo cellulare, quali ad esempio i tumori.

\subsection{La iperpolarizzazione dei nuclei per MRI tramite Dynamic Nuclear Polarization (DNP)}

Come già spiegato, la NMR e la MRI sfruttano i processi di eccitazione e successivo ritorno all'equilibro della Magnetizzazione Nucleare $(M)$. La $M$ di equilibrio lungo $z, M_{0}$ è una quantità risultante dalla somma di tutti i momenti magnetici di spin nucleari del campione per unità di volume, $i$ quali in oggetti macroscopici sono generalmente dell'ordine della mole, pari a $6,022 \times 10^{23}$. Tuttavia, $M_{0}$ dipende non solo dal numero di spin presenti ma anche dalla temperatura $(T)$ e dal campo magnetico $(H)$. Per capire ciò è necessario ricordare che in presenza di $\mathrm{H}$ i livelli energetici degli spin nucleari sono quantizzati e gli spin dei protoni, in particolare, possono acquisire una componente parallela, sul livello energetico più basso $\mid+>$, o antiparallela ad $H$, sul livello energetico più alto alto $\mid->$. La separazione energetica tra i livelli $\mid+>$ e $\mid->$, detto splitting Zeeman, è pari a $\gamma \hbar H$ che per i protoni ad 1 Tesla è circa $0.2 \mu \mathrm{eV}$, mentre a $T$ ambiente l'energia termica è circa 25 
meV. Pertanto le eccitazioni termiche tendono a rendere pressoché uguali le popolazioni dei due livelli di spin dei protoni, $N_{+}$e $N_{-}$nelle condizioni di normale operatività della MRI. Fa eccezione solo una piccolissima frazione di spin sul totale che risulta più abbondante nel livello ad energia più bassa che si definisce quindi polarizzazione nucleare:

$$
\mathrm{P}_{\mathrm{N}}=\frac{\mathrm{N}_{+}-\mathrm{N}_{-}}{\mathrm{N}_{+}+\mathrm{N}_{-}}=\tanh \left(\frac{\gamma_{\mathrm{N}} \hbar \mathrm{H}}{2 \mathrm{k}_{\mathrm{B}} \mathrm{T}}\right)
$$

dell'ordine di $1 \times 10^{-5}$ per i protoni a $T$ ambiente ed a campi in uso nell'MRI clinico. La $M$ che genera il segnale MRI, al massimo di grandezza pari a $M_{0}=\rho \gamma \hbar I P_{N}$ dove $\rho$ è la spin density ed $I$ è il numero quantico di spin nucleare pari ad $1 / 2$ per i protoni, è di riflesso altrettanto piccola: da ciò deriva la limitata sensibilità della MRI convenzionale già discussa nel paragrafo precedente.

Al fine di superare tale limite intrinseco, negli ultimi decenni sono state studiate molte tecniche dette di Iperpolarizzazione, che inducono un incremento forzato di $P_{N}$ rispetto al valore di equilibrio (Fig. 14). Tra queste la Brute Force, che prevede l'incremento della $P_{N}$ tramite un fortissimo abbassamento della $T$ e l'applicazione di $H$ molto intensi, la Spin Exchange Optical Pumping (SEOP) e la polarizzazione indotta a partire dallo stato di singoletto del Paraidrogeno (Nikolaou 2015). Le prime due sono particolarmente indicate per iperpolarizzare i gas come il ${ }^{129} \mathrm{Xe}$, utilizzabili ad esempio per l'imaging dei polmoni. La terza richiede reazioni o scambi chimici particolari e di conseguenza la sua applicazione in ambito biologico è stata limitata a combinazioni selezionate di composti catalizzatori e substrati molecolari.

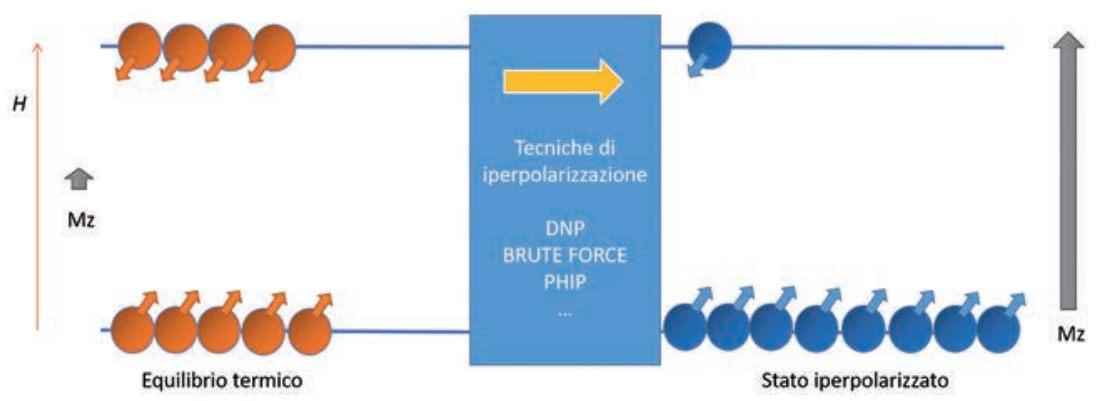

Fig. 14. Esempio illustrativo dell'accrescimento forzato della polarizzazione e della magnetizzazione nucleare tramite le tecniche di iperpolarizzazione. 
Viceversa, nell'ottica delle applicazioni in vivo una tecnica molto potente e versatile è la Dynamic Nuclear Polarization (DNP), oggetto di molti studi all'Università di Pavia da circa una decina di anni. Essa è stata ampiamente sperimentata da molti gruppi di ricerca nel mondo per iperpolarizzare molecole di interesse biologico, per lo più labellate con ${ }^{13} \mathrm{C}$, le quali vengono iniettate in un animale od un paziente e vengono successivamente analizzate con tecniche di MRI, quali la MRS o il chemical shift imaging. Le molecole iperpolarizzate generano un segnale fino a 10.000 volte più elevato di quelle presenti nel corpo (Ardenkjær-Larsen 2003) e quindi sono ben distinguibili. Inoltre, esse possono partecipare ai processi metabolici che avvengono nei tessuti, in particolare in quelli malati. Alcune pionieristiche pubblicazioni del settore (Golman 2006) e successivi studi anche a livello clinico (si veda ad esempio Wang 2019) hanno mostrato che, seguendo in tempo reale la produzione dei metaboliti derivati dal piruvato labellato con ${ }^{13} \mathrm{C}$ ed iperpolarizzato, si può localizzare con precisione un tumore, poiché in esso si riscontra una produzione di lattato anomala rispetto alle regioni sane circostanti, con ricadute importanti sulla diagnosi precoce.

L'applicazione della DNP-MRI offre molti vantaggi ma è per molti aspetti delicata. E'necessario disporre di una strumentazione nei pressi del magnete MRI per iperpolarizzare le molecole a $T$ dell'ordine di pochi gradi Kelvin e $H$ dell'ordine di alcuni Tesla, dissolverle e trasferire la soluzione nel corpo del paziente (Comment 2007). Inoltre, trasferimento ed acquisizione devono avvenire molto rapidamente, in quanto il segnale delle molecole decade per effetto del $T_{1}$ in decine di secondi. Infine, la produzione di substrati iperpolarizzati tramite DNP è oggetto di una intensa attività volta a comprendere come i parametri fisici e chimici dei materiali e le condizioni con cui la tecnica viene applicata incidano sui processi di polarizzazione e di rilassamento.

I materiali tipicamente realizzati per la DNP sono miscele costituite dalle molecole substrato da iperpolarizzare e da radicali, dispersi in piccola concentrazione rispetto al substrato. Ciò garantisce che le interazioni tra $\mathrm{i}$ radicali siano deboli e, d'altra parte, che ogni radicale abbia nel suo intorno un gran numero di spin nucleari. I radicali con un elettrone spaiato in queste condizioni sono caratterizzati da una risonanza paramagnetica elettronica $(E P R)$ stretta ed è possibile indurre un trasferimento di polarizzazione tra elettroni del radicale e nuclei della molecola substrato. Tale processo è estremamente vantaggioso: se si valuta la polarizzazione elettronica utilizzando la stessa formula (3.1), si trova infatti che, per $H$ intensi e $T$ di pochi $\mathrm{K}$, essa è pari a migliaia di volte quella nucleare, avvicinandosi al $100 \%$. 
Per trasferire la polarizzazione dagli elettroni ai nuclei il campione è irraggiato con una sorgente a microonde (MW) accordata alle frequenze tipiche della riga EPR dei radicali. A seconda dei campioni in linea di principio diversi scenari sono realizzabili (Wenckebach 2016). Grazie all'interazione dipolare elettrone-nucleo ed, in particolare, quando gli elettroni sono debolmente accoppiati tra loro dall'interazione dipolare, le MW possono polarizzare i nuclei eccitando transizioni combinate di un elettrone ed un nucleo vicini tra loro, i quali formano un piccolo sistema caratterizzato da livelli <e,nl (Fig. 15A). In genere la riga EPR in questi casi è talmente stretta che la sua larghezza di riga è inferiore a $\omega_{N}$ (Fig. 15B-1). Le transizioni rispettano il principio di conservazione dell'energia e avvengono per frequenze pari esattamente a $\omega_{M W}=\omega_{e}-\omega_{N}$, generando un processo di flip-flop $|\uparrow, \downarrow\rangle \leftrightarrow|\downarrow, \uparrow\rangle$ (Fig. 15B-2) o a $\omega_{M W}=\omega_{e}+\omega_{N}$, generando un processo di flip- flip $|\uparrow, \uparrow\rangle \leftrightarrow|\downarrow, \downarrow\rangle$. Una volta che il nucleo vicino all'elettrone irraggiato transisce, $P_{N}$ si propaga successivamente nel campione per il fenomeno detto spin diffusion promosso dall' interazione dipolare nucleo-nucleo (Fig. 15B-3). Infine, poiché gli elettroni hanno un $T_{1}$ molto più corto dei nuclei e ritornano rapidamente all'equilibrio, a seguito dell'irraggiamento ci sono sempre elettroni disponibili per fare transire i nuclei e accrescere man mano la polarizzazione nucleare (Fig. 15B-4), portando un gran numero di nuclei selettivamente in uno dei due stati di spin (Fig. 15C). Tale regime è detto di solid effect.

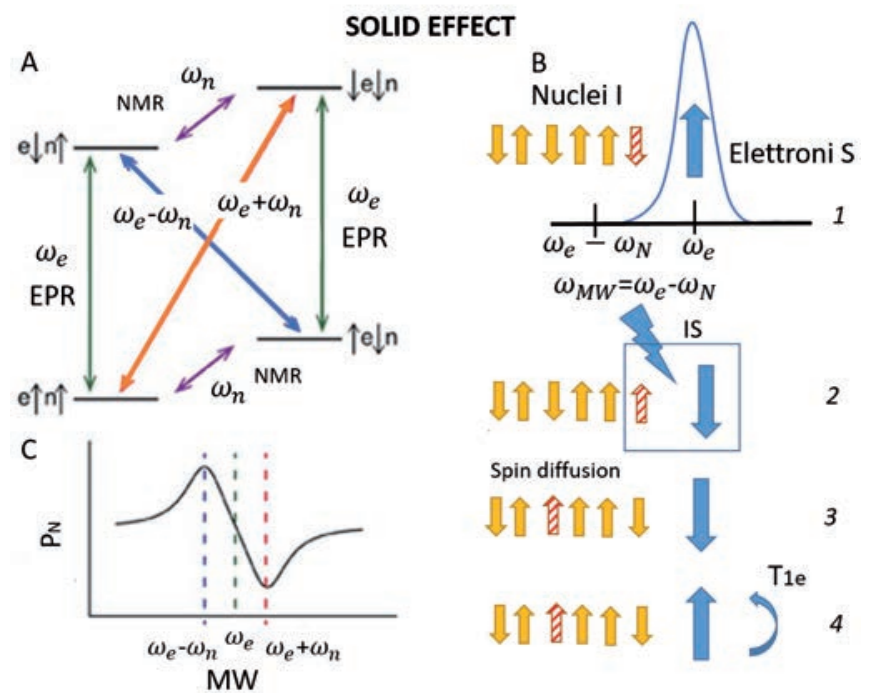

Fig. 15. Schema descrittivo dei processi in atto nel regime del Solid Effect (descritto nel testo). 
Alternativamente, se gli accoppiamenti dipolari interni ai sistemi di spin, rispettivamente quello elettronico e quello nucleare, sono forti e la larghezza di riga EPR è dell'ordine della separazione energetica tra i livelli di spin nucleari indotta da H (Fig. 16D-1), le MW non eccitano transizioni che coinvolgono singole coppie elettrone-nucleo. Si può spiegare la polarizzazione come risultato di scambi energetici tra gli ensemble degli spin nucleari ed elettronici, ognuno caratterizzato da accoppiamenti interni spin-spin che avvengono su scale di tempi molto più veloci di $T_{1}$ e tipicamente dell'ordine di $T_{2}$.

Gli accoppiamenti spin-spin interni ai due ensemble sono responsabili di uno sparpagliamento dei livelli $\mid+>$ e $\mid->$ in tanti sottolivelli con una separazione fine, dell'ordine dell'interazione dipolare (Fig. 16A). In condizioni di equilibrio termico con il reticolo, i profili di popolazione dei livelli di spin elettronici separati da $H$ e dall'interazione dipolare sono determinati entrambi dalla distribuzione di Boltzmann. Tuttavia, irraggiando con le MW una porzione selezionata della riga EPR a energie $\omega_{e}^{-} \delta$ e parificando man mano le popolazioni dei livelli energetici ad essa relativi, si crea in realtà anche un'alterazione delle popolazioni nei sotto livelli generati dall'interazione dipolare (Fig. 16B). Tradotto in termini di riserve energetiche (Fig. 16C), mentre la riserva Zeeman elettronica caratterizzata da energie dell'ordine di $\hbar \omega_{e}$ complessivamente si scalda, la riserva dipolare non-Zeeman si raffredda rispetto alla $T$ di equilibrio del reticolo.

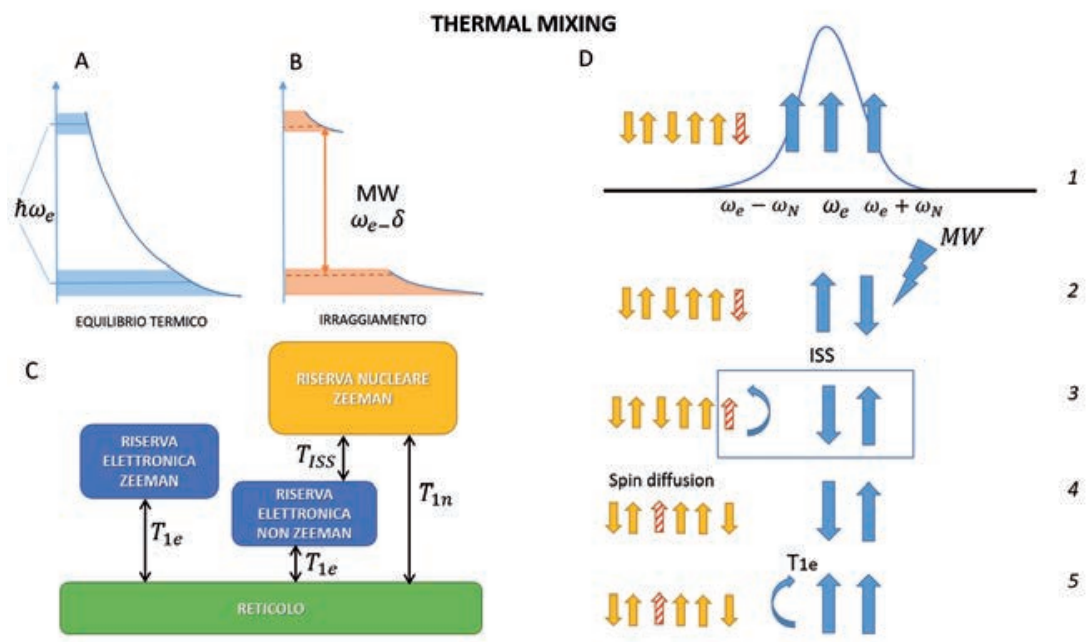

Fig. 16. Schema descrittivo dei processi in atto nel regime del Thermal Mixing (descritto nel testo). 
Infine, poichè la riserva dipolare non-Zeeman elettronica ha energie dell'ordine dello splitting Zeeman nucleare, se esiste un contatto termico efficiente tra le due riserve, anche la riserva Zeeman nucleare può raffreddarsi ed assumere una $T$ di spin più bassa della temperatura del reticolo, in altri termini, una polarizzazione più elevata di quella di equilibrio. Tale regime è detto di thermal mixing e l'interazione elettrone-nucleo chiave del processo è detta ISS (Fig. 16D-1,2,3). Questa fa sì che due elettroni separati di $\hbar \omega_{n}$ nella riserva non-Zeeman elettronica riescano a fare transire anche un nucleo a loro vicino grazie ad un loro flip-flop, in tempi caratteristici $T_{\text {ISS }}$ molto più rapidi dei $T_{1}$ nucleari ed elettronici. La massima efficienza di tale transizione avviene per $\omega_{M W} \cong$ $\omega_{e} \bar{\mp} \omega_{N}$, ma grazie alla diffusione spettrale elettronica, tutti gli elettroni della riga EPR collaborano al processo. Successivamente la diffusione di spin nucleare propaga la polarizzazione agli altri nuclei (Fig. 16D-4) ed il rilassamento spin-reticolo fa transire gli elettroni nello stato di partenza (Fig. 16D-5). Qualunque sia il regime in atto, sotto irraggiamento MW $P_{N}$ si costruisce con un tempo di polarizzazione $T_{p o l}$ che, se il processo è efficiente, è più corto del $T_{1}$ dei nuclei. Quest'ultimo risulta quindi il parametro più lungo in gioco.

All'Università di Pavia è stato studiato approfonditamente il regime fisico che si verifica in miscele tipiche di molecole e radicali per dissolution-DNP, ad esempio in campioni vetrificati di acido piruvico labellato con ${ }^{13} \mathrm{C}$ e radicali di tritile. In tali radicali la riga EPR è allargata dall'anisotropia del tensore $g$ e permette la polarizzazione dei nuclei di ${ }^{13} \mathrm{C}$. Per concentrazioni di radicali omogenee nel campione e tali da garantire un rapporto tra nuclei ed elettroni pari a 1000, gli andamenti dei $T_{1}$ nucleari ed elettronici e di $T_{p o l}$ sotto i $4 \mathrm{~K}$ sono compatibili con il regime di thermal mixing in condizioni di contatto ISS molto efficiente, che permette di raggiungere una $P_{N}$ del ${ }^{13} \mathrm{C}$ pari al $16 \%$, mentre quella all'equilibro termico sarebbe pari al $0.05 \%$ E'stato inoltre evidenziato per la prima volta il ruolo della dinamica vetrosa interna ai campioni nella determinazione dei processi di rilassamento spin-reticolo e del thermal mixing (Filibian 2014).

Studi teorici condotti parallelamente a quelli sperimentali hanno anche messo in luce l'inadeguatezza di modelli già disponibili dagli anni '60 (Abragam 1982) a spiegare la complessa fenomenologia del thermal mixing ed introdotto opportune modifiche per tenere in conto tutti i processi efficaci nella iperpolarizzazione dei nuclei, attribuendo loro grandezze realistiche, ad esempio un tempo di contatto 
$T_{\text {ISS }}$ finito, la spectral diffusion nella riga EPR conservativa o dissipativa, la leakage e la spin diffusion nucleare (Colombo Serra 2014, Caracciolo 2016). Ciò ha permesso di compiere notevoli miglioramenti e rendere i modelli maggiormente predittivi nei riguardi delle performance di polarizzazione.

Sono stati anche studiati nuovi metodi di preparazione dei materiali per DNP ottenuti a partire da substrati solidi, come ad esempio zuccheri di trealosio, miscelati a radicali TEMPO. Questi ultimi radicali, a differenza del tritile, hanno una larghezza di riga EPR adeguata a polarizzare via thermal mixing anche i protoni fino a livelli dell'ordine del $10 \%$. Lo studio di processi di polarizzazione dei protoni ha messo in luce le grandi potenzialità di tali preparazioni per potenziali applicazioni in vivo, basate su un maggior controllo dei parametri chimici e fisici dei campioni ottenuti nel processo di amorfizzazione rispetto a quanto avviene nelle soluzioni (Elisei 2015, Filibian 2016).

Più recentemente sono stati inoltre analizzati campioni solidi di ciclodestrine drogate con radicali TEMPO. Anche in questa tipologia di composti è stato possibile raggiungere una significativa $P_{N}$ dei protoni attorno al $10 \%$ a $1.6 \mathrm{~K}$ (Caracciolo 2017). Esperimenti più particolareggiati hanno messo in luce anche la dipendenza del processo di polarizzazione dalla presenza di gruppi metilici sostituiti con ${ }^{13} \mathrm{C}$ (Caracciolo 2018) e dalla sostituzione dei protoni con deuteri (Caracciolo 2019). La $P_{N}$ elevata del ${ }^{13} \mathrm{C}$ pari a $10 \%$ a $1.6 \mathrm{~K}$ ed un $T_{1}$ di circa $30 \mathrm{~s}$ a $T$ ambiente rendono questi ultimi composti molto promettenti per le successive applicazioni di dissolution DNP in vivo.

\subsection{Il nuovo centro di Imaging preclinico dell'Università degli Studi di Pavia}

Il Laboratorio di MRI e Microtomografia a Raggi X (microCT) è di recentissima costituzione ed è adiacente ai locali del Centro di servizio per la gestione unificata delle attività di stabulazione e di radiobiologia del Palazzo Golgi Spallanzani dell'Università di Pavia. Le sue attività sono state avviate nell'autunno del 2019 grazie all'ingente finanziamento pervenuto ai Dipartimenti di Fisica e di Medicina Molecolare nell'ambito del bando nazionale 'Dipartimenti di Eccellenza' dell'anno 2017 ed al cofinanziamento di Ateneo mediante il Piano di Acquisizione di Strumentazioni Scientifiche (PASS).

Il laboratorio effettua i servizi di una facility rivolta ad utenti interni 
ed esterni ed è gestita dal Centro Grandi Strumenti di Ateneo. Gli esperimenti MRI vengono effettuati tramite un tomografo Bruker PharmaScan 70/16 US, con campo magnetico di 7 Tesla (Figg. 10 e 17A) e quelli di microCT tramite uno scanner Sky Scan 1276 Bruker ad alta risoluzione (Fig. 17A-B). Entrambi gli strumenti sono equipaggiati per effettuare esperimenti in vivo, essendo presenti apparecchiature per anestesia gassosa (Fig. 17C) e dotazioni per varie tipologie di analisi, ma possono anche essere utilizzati per ricerche avanzate in altri ambiti quali, ad esempio, la scienza dell'alimentazione e dei materiali.

Il Laboratorio costituisce a tutti gli effetti una piattaforma multimodale per condurre studi avanzati di Imaging. In particolare, esso è dotato di una strumentazione unica nel suo genere che permette di condurre le tomografie MRI e $\mu \mathrm{CT}$ in modo sequenziale sullo stesso campione. Ciò è estremamente vantaggioso in quanto, tramite le due tecniche si ottengono informazioni in molti casi complementari.
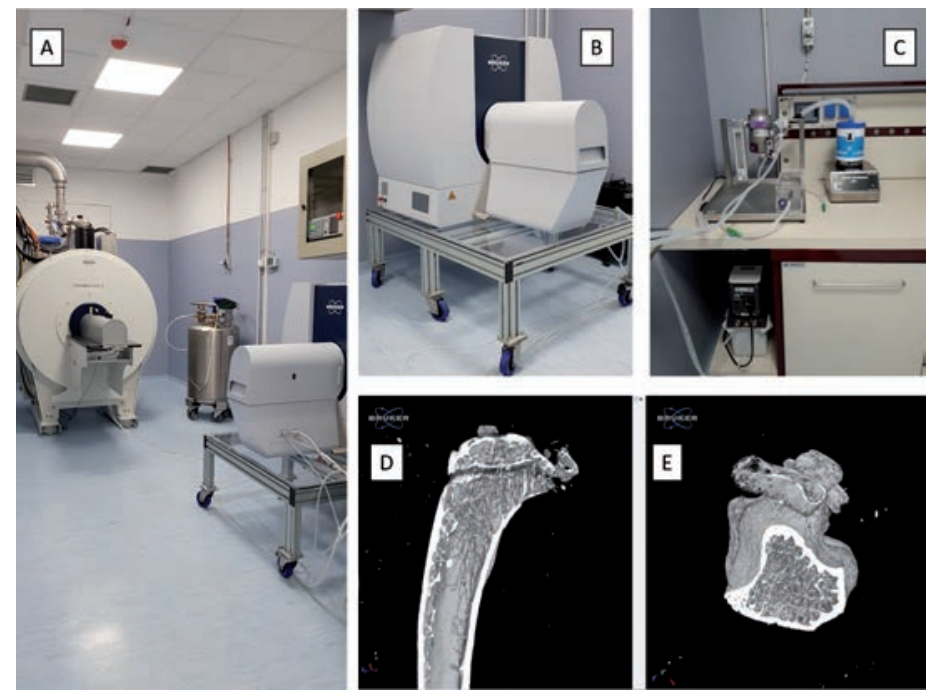

Fig. 17. Immagini della facility di Imaging preclinico dell'Università degli Studi di Pavia $(A-B-C)$ ed esempi di sezioni da ricostruzioni $3 D$ di una tibia di topo viste da varie prospettive (D-E). La risoluzione spaziale di ben 4 micron rende ben visibili le strutture trabecolari interne all'osso (R. Besio, Univ. Pavia).

Rispetto alla MRI, descritta estesamente nel paragrafo 3.1, la microCT è in grado di fornire ricostruzioni 3D non distruttive delle strutture interne ad alta risoluzione, basate su voxel con lato riducibile 
fino a $2.8 \mu \mathrm{m}$. Poiché la microCT sfrutta l'attenuazione dei raggi $\mathrm{X}$ da parte dei materiali, essa è la tecnica d'elezione per lo studio di materiali densi, quali le ossa, come mostrato in Fig. 17D-E, in combinazione alle tradizionali analisi genetiche e istologiche. L'utilizzo di mezzi di contrasto, tuttavia, estende l'uso dello strumento all'analisi non invasiva di vari tessuti molli quali, ad esempio, il polmone ed il sistema vascolare. Con la microCT è possibile ottenere quindi misurazioni precise della micro-architettura delle strutture interne ed una dettagliata visualizzazione, utile ad essere coregistrata alle immagini di MRI in grado di fornire, d'altra parte, un'ampia varietà di parametri funzionali e metabolici dei tessuti.

La strumentazione acquisita dal Laboratorio di Imaging preclinico permetterà un'ulteriore espansione degli studi già in atto all'Università di Pavia su agenti di contrasto e nanoparticelle magnetiche, permettendo studi in vitro a campo alto e test in vivo. Inoltre, è sicuramente una risorsa preziosa per approfondire l'iperpolarizzazione di molecole di interesse biomedico e per trovare metodi avanzati e più efficienti di trasferimento delle molecole dal sito polarizzatore fino all'interno del magnete MRI. Si prevede di potenziare a breve il laboratorio con strumentazione per imaging ottico e per l'ipertermia magnetica.

\section{RINGRAZIAMENTI}

Si ringraziano il Dott. Marco Peviani (Univ. Pavia) per aver concesso l'utilizzo delle Figg. 11 e 12, il Dott. Davide Cicolari ed il Prof. Alessandro Lascialfari (Univ. Pavia) per la Fig. 13 e la Dott.ssa Roberta Besio e la Prof.ssa Antonella Forlino (Univ. Pavia) per la Fig. 17D-E.

Le Figg. 10, 11, 12, 13 e 17 sono state ottenute grazie ai finanziamenti “MIUR Dipartimenti di Eccellenza 2018-2022" attribuiti rispettivamente ai Dipartimenti di Fisica e di Medicina Molecolare dell'Università di Pavia. Inoltre i risultati riportati in Figg. 11 e 12 sono stati finanziati da ALSA grant nr. 20-IIA-525. 


\section{BIBLIOGRAFIA}

Abragam 1982 - A. Abragam and M. Goldman, Nuclear magnetism: order and disorder, Oxford, Clarendon Press, 1982.

Akhtar 2021 - K. Akhtar, Y. Javed, M. I. Akhtar and N. A. Shad, Applications of Iron Oxide Nanoparticles in the Magnetic Resonance Imaging for the Cancer Diagnosis, in V. Yata, S. Ranjan, N. Dasgupta and E. Lichtfouse (editors), Nanopharmaceuticals: Principles and Applications Vol. 1, Springer, Cham., 2021, 115-158.

Alexander 2007 - A. L. Alexander, J. E. Lee, M. Lazar and A. S. Field, Diffusion tensor imaging of the brain, Neurotherapeutics 4 (2007), 316-329.

Ardenkjær-Larsen 2003 - J. H. Ardenkjær-Larsen, B. Fridlund, A. Gram, G. Hansson, L. Hansson, M. H. Lerche, R. Servin, M. Thaning and K. Golman, Increase in signal-to-noise ratio of $>$ 10,000 times in liquid-state NMR, PNAS 100 (2003), 10158-10163.

Basini 2019 - M. Basini, A. Guerrini, M. Cobianchi, F. Orsini, D. Bettega, M. Avolio, C. Innocenti, C. Sangregorio, A. Lascialfari and P. Arosio, Tailoring the magnetic core of organic-coated iron oxides nanoparticles to influence their contrast efficiency for Magnetic Resonance Imaging, J. Alloys Compd. 770 (2019), 58-66.

Bednorz 1986 - J. G. Bednorz and K. A. Müller, Possible bigh T superconductivity in the Ba-La-Cu-O system, Z. Physik B-Condensed Matter 64 (1986), 189-193.

Bernstein 2004 - M. A. Bernstein, K. F. King and X. J. Zhou, Handbook of MRI pulse sequences, Burlington, MA, Elsevier Academic Press, 2004.

Bloch 1946 - F. Bloch, W. W. Hansen and M. Packard, The nuclear induction experiment, Phys Rev 70 (1946), 474-485.

Bogani 2008 - L. Bogani and W. Wernsdorfer, Molecular spintronics using single-molecule magnets, Nature Mater 7 (2008), 179-186.

Borsa 2006 - F. Borsa, A. Lascialfari and Y. Furukawa, NMR in Magnetic Molecular Rings and Clusters, in J. Dolinšek, M. Vilfan and S. Žumer (editors), Novel NMR and EPR techniques (Lecture Notes in Physics, 684), Berlin, Heidelberg, Springer, 2006, 297-349.

Borsa 2007 - F. Borsa, NMR in Magnetic Single Molecule Magnets, in P. Carretta and A. Lascialfari (editors), NMR-MRI, $\mu S R$ and Mössbauer Spectroscopies in Molecular Magnets, Milano, Springer, 2007, 29-70.

Bossoni 2013 - L. Bossoni, P. Carretta, M. Horvatić, M. Corti, A. Thaler and P. C. Canfield, Glassy transition in the vortex lattice of $\mathrm{Ba}\left(\mathrm{Fe}_{0.93} \mathrm{R} \mathrm{h}_{0.07}\right)_{2} \mathrm{As} \mathrm{s}_{2}$ superconductor probed by NMR and ac-susceptibility, Europhys. Lett. 102 (2013), 17005.

Branzoli 2009 - F. Branzoli, P. Carretta, M. Filibian, G. Zoppellaro, M. J. Graf, J. R. Galan-Mascaros, O. Fuhr, S. Brink and M. Ruben, Spin dynamics in the negatively charged terbium (III) bis-phthalocyaninato complex, J. Am. Chem. Soc. 131 (2009), 4387-4396.

Brero 2020 - F. Brero, M. Basini, M. Avolio, F. Orsini, P. Arosio, C. Sangregorio, C. Innocenti, A. Guerrini, J. Boucard, E. Ishow, M. Lecouvey, J. Fresnais, L. Lartigue and A. Lascialfari, Coating Effect on the ${ }^{1} \mathrm{H}-\mathrm{NMR}$ Relaxation Properties of Iron Oxide Magnetic Nanoparticles, Nanomaterials 10 (2020), 1660. Caracciolo 2016 - F. Caracciolo, M. Filibian, P. Carretta, A. Rosso and A. De Luca, 
Evidence of spin-temperature in dynamic nuclear polarization: an exact computation of the EPR spectrum, Phys. Chem. Chem. Phys. 18 (2016), 25655-25662.

Caracciolo 2017 - F. Caracciolo, P. Carretta, M. Filibian and L. Melone, Dynamic Nuclear Polarization of $\beta$-Cyclodextrin Macromolecules, J. Chem. Phys. B, 121 (2017), 2584-2593.

Caracciolo 2018 - F. Caracciolo, A. Lucini Paioni, M. Filibian, L. Melone, and P. Carretta, Proton and Carbon-13 Dynamic Nuclear Polarization of Metbylated $\beta$ Cyclodextrins, Phys. Chem. B 122 (2018), 1836-1845.

Caracciolo 2019 - F. Caracciolo, E. Charlaftis, L. Melone, and P. Carretta, Molecular Dynamics and Hyperpolarization Performance of Deuterated B-Cyclodextrins, J. Phys. Chem. B 123 (2019), 3731-3737.

Carretta 2020 - P. Carretta and G. Prando, Iron-based superconductors: tales from the nuclei, Riv. Nuovo Cim. 43 (2020), 1-43.

Casula 2010 - M. F. Casula, P. Floris, C. Innocenti, A. Lascialfari, M. Marinone, M. Corti, R. A. Sperling, W. J. Parak and C. Sangregorio, Magnetic resonance imaging contrast agents based on iron oxide superparamagnetic ferro fluids, Chem. Mater. 22 (2010), 1739-1748.

Colombo Serra 2014 - S. Colombo Serra, M. Filibian, P. Carretta, A. Rosso and F. Tedoldi, Relevance of electron spin dissipative processes to dynamic nuclear polarization via thermal mixing, Phys. Chem. Chem. Phys. 16 (2014), 753-764.

Comment 2007 - A. Comment, B. van den Brandt, K. Uffmann, F. Kurdzesau, S. Jannin, J. A. Konter, P. Hautle, W. Th. Wenckebach, R. Gruetter and J. J. van der Klink, Design and performance of a DNP prepolarizer coupled to a rodent MRI scanner, Concepts Magn. Reson. 31B (2007), 255-269.

Corti 1996 - M. Corti, B. J. Suh, F. Tabak, A. Rigamonti, F. Borsa, M. Xu and B. Dabrowski, Flux-line dynamics in $\mathrm{YBa}_{2} \mathrm{Cu}_{4} \mathrm{O}_{8}$ from ${ }^{89} \mathrm{Y}$ NMR, Phys Rev B 54 (1996), 9469-9474.

Damadian 1971 - R. Damadian, Tumor Detection by Nuclear Magnetic Resonance, Science 171 (1971), 1151-1153.

Deruelle 2020 - T. Deruelle, F. Kober, A. Perles-Barbacaru, T. Delzescaux, V. Noblet, E. L. Barbier and M. Dojat, A Multicenter Preclinical MRI Study: Definition of Rat Brain Relaxometry Reference Maps, Front Neuroinform. 14:22 (2020).

Dey 2018 - A. Dey, P. Kalita and V. Chandrasekhar, Lanthanide(III)-Based Single-Ion Magnets, ACS Omega 3 (2018), 9462-9475.

Duarte 2012 - J. M. N. Duarte, H. Lei, V. Mlynárik and R. Gruetter, The neurochemical profile quantified by in vivo ${ }^{1} H$ NMR spectroscopy, NeuroImage 61 (2012), 342-362.

Elisei 2015 - E. Elisei, M. Filibian, P. Carretta, S. Colombo Serra, F. Tedoldi, J. F. Willart, M. Descamps and A. Cesàro, Dynamic nuclear polarization of a glassy matrix prepared by solid state mechanochemical amorphization of crystalline substances, Chem. Commun. 51 (2015), 2080-2083.

Filibian 2012 - M. Filibian, A. Frasca, D. Maggioni, E. Micotti, A. Vezzani and T. Ravizza, In vivo imaging of glia activation using ${ }^{1} \mathrm{H}$ magnetic resonance spectroscopy to detect putative biomarkers of tissue epileptogenicity, Epilepsia 53 (2012), 1907-1916.

Filibian 2014 - M. Filibian, S. Colombo Serra, M. Moscardini, A. Rosso, F. Tedoldi and 
P. Carretta, The role of the glassy dynamics and thermal mixing in the dynamic nuclear polarization and relaxation mechanisms of pyruvic acid, Phys. Chem. Chem. Phys. 16 (2014), 27025-27036.

Filibian 2016 - M. Filibian, E. Elisei, S. Colombo Serra, A. Rosso, F. Tedoldi, A. Cesàro and P. Carretta, Nuclear magnetic resonance studies of DNP-ready trebalose obtained by solid state mechanochemical amorphization, Phys. Chem. Chem. Phys. 18 (2016), 16912-16920.

Fukushima 1981 - E. Fukushima and S. B. W. Roeder, Experimental Pulse NRM: A Nuts and Bolts Approach, Reading, MA, Addison-Wesley, 1981.

Gatteschi 2011 - D. Gatteschi, R. Sessoli and J. Villain, Molecular Nanomagnets, Oxford, Oxford Univ. Press, 2011.

Golman 2006 - K. Golman, R. in't Zandt and M. Thaning, Real-time metabolic imaging, Proc Natl Acad Sci U S A 103 (2006), 11270-11275; K. Golman, R. in't Zandt, M. Lerche, R. Pehrson and J. H. Ardenkjaer-Larsen, Metabolic Imaging by Hyperpolarized ${ }^{13} \mathrm{C}$ Magnetic Resonance Imaging for In vivo Tumor Diagnosis, Cancer Res 66 (2006), 10855-10860.

Gorter 1942 - C. J. Gorter and L. J. F. Broer, Negative result of an attempt to observe nuclear magnetic resonance in solids, Physica 9 (1942), 591-596.

Hahn 1950 - E. L. Hahn, Spin echoes, Phys. Rev. 80 (1950), 580-594.

Hartung 2011 - M. P. Hartung, T. M. Grist and C. J. François, Magnetic resonance angiography: current status and future directions, J Cardiovasc Magn Reson 13:19 (2011).

Henning 1986 - J. Hennig, A. Nauerth and H. Friedburg, RARE imaging - a fast imaging method for clinical MR, Magn Reson Med 3 (1986), 823-833.

Herborn 2012 - C. U. Herborn, Contrast Agents for MR Angiography, in J. Carr and T. Carroll T. (editors), Magnetic Resonance Angiography, New York, Springer, 2012.

Ho 2012 - M.-L. Ho, R. Rojas, and R. L. Eisenberg, Cerebral Edema, American Journal of Roentgenology 199 (2012), 258-273.

Horská 2010 - A. Horská and P. B. Barker, Imaging of brain tumors: MR spectroscopy and metabolic imaging, Neuroimaging Clin N Am. 20 (2010), 293-310.

Ishikawa 2003 - N. Ishikawa, M. Sugita, T. Okubo, N. Tanaka, T. Iino and Y. Kaizu, Determination of ligand-field parameters and f-electronic structures of doubledecker bis(phthalocyaninato)lanthanide complexes, Inorg. Chem. 42 (2003), 2440-2446; N. Ishikawa, M. Sugita, T. Ishikawa, S.Y. Koshihara and Y. Kaizu, Lanthanide double-decker complexes functioning as magnets at the single-molecular level, J Am Chem Soc. 125 (2003), 8694-8695.

Jeong 2018 - Y. Jeong, H. S. Hwang and K. Na, Theranostics and contrast agents for magnetic resonance imaging, Biomater Res 22:20 (2018).

Ladd 2018 - M. E. Ladd, P. Bachert, M. Meyerspeer, E. Moser, A. M. Nagel, D. G. Norris, S. Schmitter, O. Speck, S. Straub and M. Zaiss, Pros and cons of ultrabigh-field MRI/MRS for buman application, Progress in Nuclear Magnetic Resonance Spectroscopy 109 (2018), 1-50.

Lascialfari 2013 - A. Lascialfari, M. Filibian, C. Sangregorio and P. Carretta, In vivo biomedical applications of magnetic resonance and magnetic materials, La Rivista del Nuovo Cimento 6 (2013), 211-271. 
Leuenberger 2001 - M. N. Leuenberger and D. Loss, Quantum computing in molecular magnets, Nature 410 (2001), 789-793.

Lis 1980 - T. Lis, Preparation, structure, and magnetic properties of a dodecanuclear mixed-valence manganese carboxylate, Acta crystallogr. B 36 (1980), 2042-2046.

Lüsebrink 2017 - F. Lüsebrink, A. Sciarra, H. Mattern and O. Speck, $T_{1}$-weighted in vivo buman whole brain MRI dataset with an ultrahigh isotropic resolution of 250 $\mu m$, Sci Data 4:170032 (2017).

Ma 2013 - D. Ma, V. Gulani, N. Seiberlich, K. Liu, J. L. Sunshine, J. L. Duerk and M. A. Griswold, Magnetic resonance fingerprinting, Nature 495 (2013),187-192.

Mandino 2020 - F. Mandino, D. H. Cerri, C. M. Garin, M. Straathof, G. A. F. van Tilborg, M. M. Chakravarty, M. Dhenain, R. M. Dijkhuizen, A. Gozzi, A. Hess, S. D. Keilholz, J. P. Lerch, Y.-Y. I. Shih and J. Grandjean, Animal Functional Magnetic Resonance Imaging: Trends and Path Toward Standardization, Front. Neuroinform, 13:78 (2020).

Marzola 2016 - P. Marzola, F. Boschi, F. Moneta, A. Sbarbati and C. Zancanaro, Preclinical In vivo Imaging for Fat Tissue Identification, Quantification, and Functional Characterization, Front. Pharmacol. 7: 336 (2016).

Mehta 2019 - B. Bipin Mehta, S. Coppo, D. Frances McGivney, J. I. Hamilton, Y. Chen, Y. Jiang, D. Ma, N. Seiberlich, V. Gulani and M. A. Griswold, Magnetic resonance fingerprinting: a technical review, Magn Reson Med. 81 (2019), 25- 46.

Moreno-Pineda 2018 - E. Moreno-Pineda, C. Godfrin, F. Balestro, W. Wernsdorfer and M. Ruben, Molecular spin qudits for quantum algorithms, Chem. Soc. Rev. 47 (2018), 501-513.

Nikolaou 2015 - P. Nikolaou, B. M. Goodson and E. Y. Chekmenev, NMR byperpolarization techniques for biomedicine, Chemistry 21 (2015), 3156-3166.

Ogawa 1990 - S. Ogawa, T. M. Lee, A. R. Kay and D. W. Tank, Brain magnetic resonance imaging with contrast dependent on blood oxygenation, Proc Natl Acad Sci USA. 87 (1990), 9868-9872.

Orlando 2016 - T. Orlando, M. Albino, F. Orsini, C. Innocenti, M. Basini, P. Arosio, C. Sangregorio, M. Corti, A. Lascialfari, On the magnetic anisotropy and nuclear relaxivity effects of $\mathrm{Co}$ and $\mathrm{Ni}$ doping in iron oxide nanoparticles, J. Appl. Phys. 119, 134301 (2016).

Öz 2014 - G. Öz et al., Clinical proton MR spectroscopy in central nervous system disorders, Radiology 270 (2041), 658-679.

Pautler 2004 - R. G. Pautler, Mouse MRI: Concepts and Applications in Physiology, Physiology 19 (2004), 168-175.

Pfeuffer 1999 - J. Pfeuffer, I. Tkác, S. W. Provencher and R. Gruetter, Toward an in vivo neurochemical profile: quantification of 18 metabolites in short-echo-time ${ }^{1} \mathrm{H}$ NMR spectra of the rat brain, J Magn Reson. 141 (1999), 104-120.

Prasad 2021 - S. Prasad, A. Chandra, M. Cavo, E. Parasido, S. Fricke, Y Lee, E. D’Amone, G. Gigli, C. Albanese, O. Rodriguez and L. L. del Mercato, Optical and magnetic resonance imaging approaches for investigating the tumour microenvironment: state-of-the-art review and future trends, Nanotechnology 32 (2021), 062001.

Rabi 1938 - I. I. Rabi, J. R. Zacharias, S. Millman and P. Kusch, A new method of measuring nuclear magnetic moment, Phys. Rev. 53 (1938), 318. 
Rigamonti 1998 - A. Rigamonti, F. Borsa and P. Carretta, Basic aspects and main results of NMR-NQR spectroscopies in bigh-temperature superconductors, Rep. Prog. Phys. 61 (1998), 1367-1439.

Rigamonti 2015 - A. Rigamonti and P. Carretta , Structure of Matter: an Introductory Course with Problems and Solutions, 3rd Edition, Ch. 16, Milano, Springer, 2015.

Saeed 2015 - M. Saeed, T. A. Van, R. Krug, S. W. Hetts and M. W. Wilson, Cardiac MR imaging: current status and future direction, Cardiovasc Diagn Ther 5 (2015), 290-310.

Serkova 2017 - N. J. Serkova, Nanoparticle-Based Magnetic Resonance Imaging on Tumor-Associated Macrophages and Inflammation, Front Immunol. 8:590 (2017).

Sessoli 1993 - R. Sessoli, D. Gatteschi, A. Caneschi and M. A. Novak, Magnetic bistability in a metal-ion cluster, Nature 365 (1993), 141-143.

Sessoli 2017 - R. Sessoli, Magnetic molecules back in the race, Nature 548 (2017), 400401.

Slichter 1990 - C. P. Slichter, Principles of Magnetic Resonance, 3rd ed., New York, Springer-Verlag, 1990.

Sneider 2020 - E. Snider, N. Dasenbrock-Gammon, R. McBride, M. Debessai, H. Vindana, K. Vencatasamy, K. V. Lawler, A. Salamat and R. P. Dias, Room-temperature superconductivity in a carbonaceous sulfur bydride, Nature 586 (2020), 373-377.

Timco 2009 - G. A. Timco, S. Carretta, F. Troiani, F. Tuna, R. J. Pritchard, C. A. Muryn, E. J. L. McInnes, A. Ghirri, A. Candini, P. Santini, G. Amoretti, M. Affronte and R. E. P. Winpenny, Engineering the coupling between molecular spin qubits by coordination chemistry, Nature Nanotech 4 (2009), 173-178.

Wang 2019 - Z. J. Wang, M. A. Ohliger, P. E. Z. Larson, J. W. Gordon, R. A. Bok, J. Slater, J. E. Villanueva-Meyer, C. P. Hess, J. Kurhanewicz and D. B. Vigneron, Hyperpolarized ${ }^{13} \mathrm{C}$ MRI: State of the Art and Future Directions, Radiology 291 (2019), 273-284.

Wenckebach 2016 - T. Wenckebach, The Principles of Dynamic Nuclear Polarisation, The Nederlands, Spindrift, 2016.

Wu 2016 - B. Wu, G. Warnock, M. Zaiss, C. Lin, M. Chen, Z. Zhou, L. Mu, D. Nanz, R. Tuura and G. Delso, An overview of CEST MRI for non-MR physicists, EJNMMI Phys 3:19 (2016).

Xiao 2016 - Y. -D. Xiao, R. Paudel, J. Liu, C. Ma, Z. -S. Zhang, and S.K. Zhou, MRI contrast agents: Classification and application (Review).), Int. J. Mol. Med. 38 (2016), 1319-1326.

Yamada 2009 - K. Yamada, K. Sakai, K. Akazawa, S. Yuen and T. Nishimura, MR tractography: a review of its clinical applications, Magn Reson Med Sci. 8 (2009), 165 174.

Zhou 2013 - Z. Zhou and Z. -R. Lu, Gadolinium-based contrast agents for magnetic resonance cancer imaging, WIREs Nanomed Nanobiotechnol 5 (2013), 1-18. 
